Article

\title{
Synthetic Route to Glycosyl $\beta-1 C$-(phosphino)- phosphonates as Unprecedented Stable Glycosyl Diphosphate Analogs and Their Preliminary Biological Evaluation
}

\author{
Michaël Bosco ${ }^{1} \oplus$, Su-Jin Paik ${ }^{2} \oplus$, Patricia Busca ${ }^{1} \oplus$, Stuart E. H. Moore ${ }^{2}(\mathbb{D}$ and \\ Christine Gravier-Pelletier 1,*iD \\ 1 Université de Paris, Faculté des Sciences, Campus Saint-Germain-des-Prés, UMR CNRS 8601, LCBPT, \\ 45 rue des Saints Pères, F-75006 Paris, France; michael.bosco@u-paris.fr (M.B.); \\ patricia.busca@u-paris.fr (P.B.) \\ 2 Université de Paris, Faculté de Médecine Xavier Bichat, INSERM U1149, CRI, 16 rue Henri Huchard, \\ F-75018 Paris, France; su-jin.paik@inserm.fr (S.-J.P.); stuart.moore@inserm.fr (S.E.H.M.) \\ * Correspondence: christine.gravier-pelletier@u-paris.fr; Tel.: +33-176-534-228
}

Academic Editor: László Somsák

Received: 2 October 2020; Accepted: 26 October 2020; Published: 27 October 2020

\begin{abstract}
The synthesis of glycosyl- $\beta-1 C$-(phosphino)-phosphonates is a challenge since it has not yet been described. In this paper, we report an innovative synthetic method for their preparation from Glc-, Man-, and GlcNAc- lactone derivatives. The proposed original strategy involves the addition of the corresponding $\delta$-hexonolactones onto the dianion of (methylphosphino) phosphonate as a key step, followed by dehydration and stereoselective addition of dihydrogen on the resulting double bond. Final deprotection provides the new glycosyl diphosphate analogs in 35\%,36\%, and $10 \%$ yield over 6 steps from the corresponding $\delta$-hexonolactones. The synthetized compounds were evaluated as inhibitors of phosphatase and diphosphatase activities and found to have complex concentration-dependent activatory and inhibitory properties on alkaline phosphatase. The synthetized tools should be useful to study other enzymes such as transferases.
\end{abstract}

Keywords: C-glycoside; diphosphate analogs; phosphinophosphonate; alkaline diphosphatase; glycochemistry

\section{Introduction}

Glycosyl diphosphates are key constituents of biologically-active compounds. In glycosylation reactions catalyzed by glycosyl transferases, monosaccharides are activated as $\alpha$ - or $\beta$-glycosyl esters of nucleotides, which are major glycosylating agents in the biosynthesis of oligosaccharides. Examples of nucleotide sugars such as UDP- $\alpha$-D-Glc, GDP- $\alpha$-D-Man, GDP- $\beta$-L-Fuc, or ADP-L-glycero- $\beta$-D-mannoheptose are shown in Figure 1. They are also involved as glycosyl donors in glycoconjugate biosynthesis [1,2] such as $N$-linked glycoproteins [3-5] resulting from the transfer of a polysaccharide from a dolichol linked oligosaccharide (DLO, Figure 1) onto polypeptides. Noteworthy, UDP-GlcNAc (Figure 1) which is involved in this biosynthetic pathway is also a key intermediate in the biosynthesis of bacterial peptidoglycan precursors [6] such as UDP-MurNAc-pentapeptide (Figure 1), the nucleotidic substrate of the enzymatic reaction catalyzed by the bacterial transferase MraY [7,8], highlighting the analogies in lipopolysaccharide and glycoprotein biosynthesis [9].

Due to their central role in carbohydrate metabolism, much attention has been paid to the development of synthetic strategies towards non-hydrolysable diphosphate analogs of nucleotide sugars, notably towards potential inhibitors of enzymes involved in glycosyl phosphate metabolism. 
UDP-Glc or UDP-GlcNAc were the most studied and research efforts led to the synthesis of several analogs with very variable structures. Representative synthetic analogs are outlined in Figure 2. On the one hand, phosphonate derivatives in which the oxygen atom in either the anomeric position of the sugar [10-12] or between the phosphorus atoms [13] has been replaced by a methylene group have been reported. On the other hand, the chain length between the sugar and the diphosphate has been increased [14,15]. In other diphosphate analogs, one of the phosphorus atoms has been replaced by a carbonyl group [16]. Various linkers lacking any phosphorus atoms have also been envisaged as diphosphate mimics such as an $\alpha, \beta$-dihydroxy ketone [17] or an oxycarbonylaminosulfonyl [18]. A $\beta$-D-glycosyl residue has also been linked to uridine in allophanates and biuret derivatives [19]. Finally, the isosteric replacement of a diphosphate by a triazole linker [20] was also described. Noteworthy, diphosphate mimics involving other sugars or bases notably include a carbonyl linked to a $\mathrm{O}$ - or $N$-sulfamoyl uridine [21], tartaric [22], saccharidic [23,24], and squaryldiamide [25] linkers.

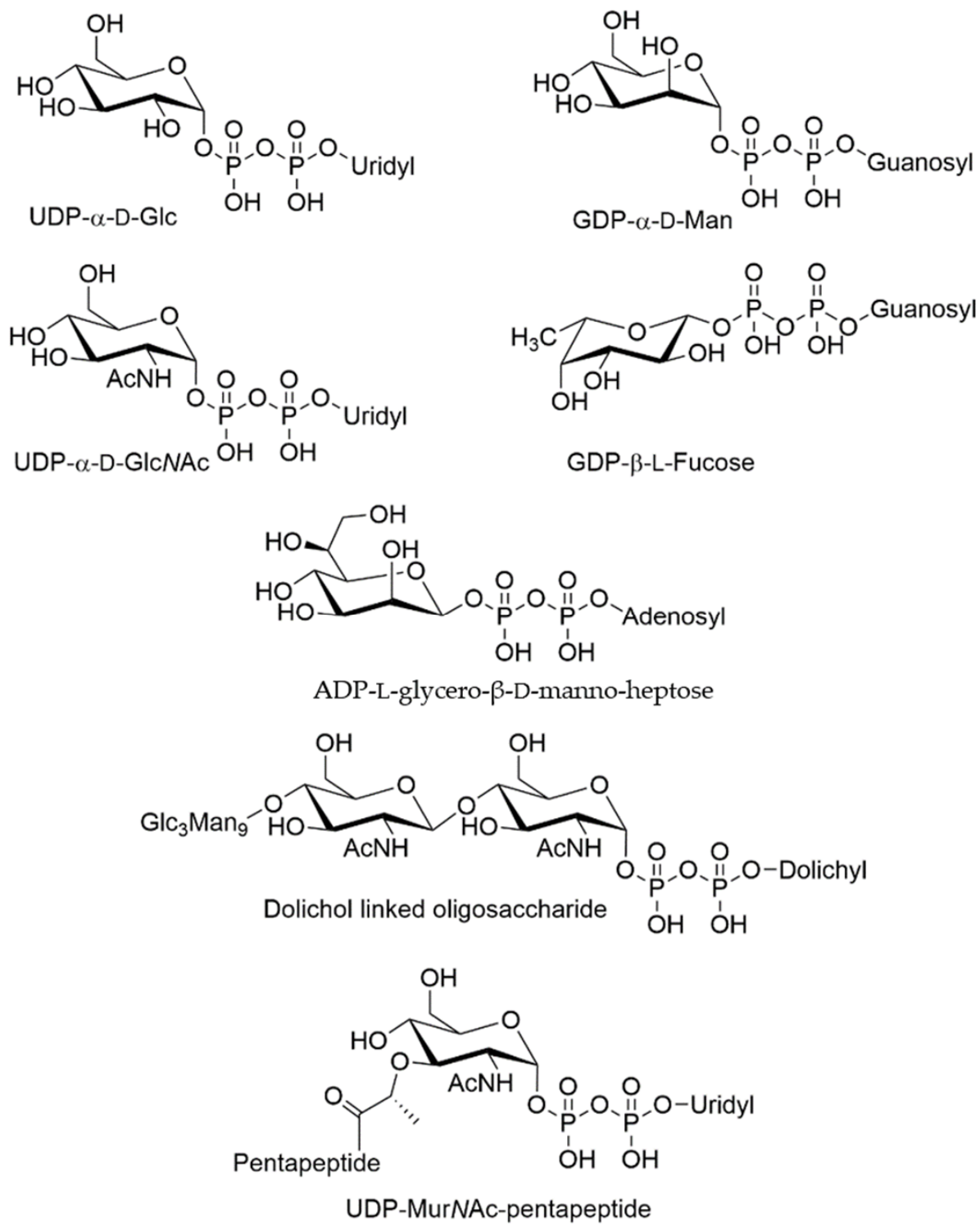

Figure 1. Biologically relevant glycosyl diphosphates. 
<smiles>O=C(O)CP(=O)([O-])OP(=O)([O-])O[Na]</smiles><smiles>O=P([O-])(O)CP(=O)([O-])OCl</smiles><smiles>O=C(OCl)C(O)P(=O)([O-])OP(=O)([O-])O[Hg]</smiles>

ref. 14<smiles>[R]C(CC(Cl)(Cl)Cl)P(=O)(O)OP(=O)([O-])OO</smiles>

$\mathrm{R}=\mathrm{OH}$, ref. 14 $\mathrm{R}=\mathrm{H}$, ref. 15

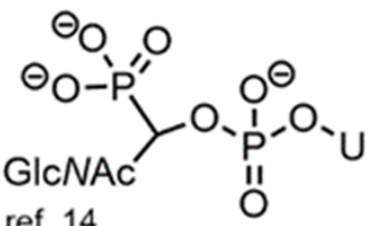

ref. 14<smiles>O=C(O)CC(=O)CP(=O)([O-])O[Hg]</smiles>

ref.16<smiles>[Y]C(=O)C(O)C(O)CC(C)(Cl)Cl</smiles>

ref.17 $\mathrm{X}=\mathrm{O}, \mathrm{NH}$<smiles></smiles>

ref. 18<smiles>[X]C(=O)NC(=O)NCl</smiles>

ref. 19

$\mathrm{X}=\mathrm{O}, \mathrm{NH}$

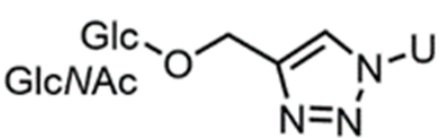

ref. 20

Figure 2. Examples of synthetic analogs of UDP-Glc or UDP-GlcNAc.

In this context, our goal was to develop a straightforward access to original analogs of glycosyl diphosphate with an unprecedented $\beta-1-C$-(phosphino)-phosphonate structure. On the one hand, the replacement of an oxygen atom at the anomeric position of a saccharidic unit by a methylene group is a well-documented strategy $[26,27]$ to prevent the cleavage of the aglycon part of the molecule during hydrolysis by glycosidases. On the other hand, the proposed diphosphate analog should have the property to chelate a divalent cation as diphosphates often do, while limiting the cleavage between the phosphorus atoms due to the poor leaving group character of a phosphonate compared to that of a phosphate. To extend the scope of the proposed method, the preparation of Man-, Glc-, and GlcNAc-diphosphate analogs has been envisaged (Figure 3).

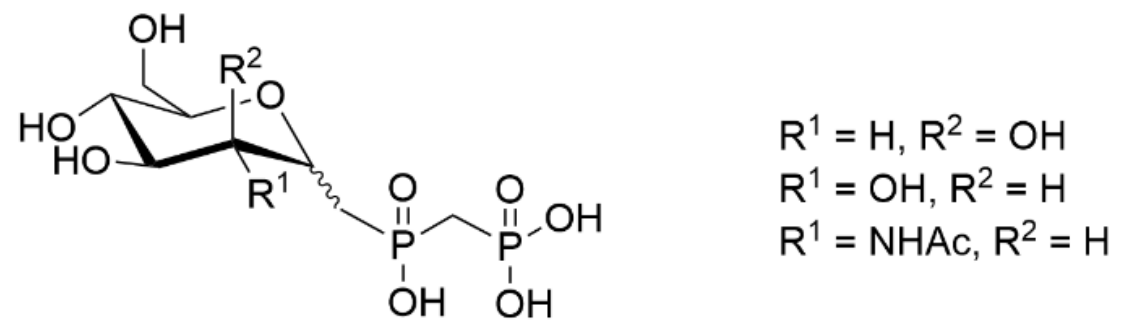

Figure 3. Structure of the targeted compounds.

The synthesis of a phosphino-phosphonate skeleton has never been described for monosaccharide derivatives. Nevertheless, this phosphorus scaffold was synthesized according to different pathways (Figure 4) by nucleophilic substitution of the chlorine atom of a farnesylchlorophosphinate by a lithio phosphonate [28] (Figure 4A) or by the Michaelis-Arbuzov reaction of a phenylphosphite on a (chloromethylene)phosphonate (Figure 4B). However, such a reaction involves rather drastic conditions [29] that might not be the most appropriate for sensitive molecules such as glycosides and would involve the sequential introduction of phosphorus derivatives on a glycosyl compound. A phospha-Claisen condensation involving the preparation of a lithio phosphonate that reacts with an (hydroxymethylene)phosphonate [30] (Figure 4C) presents the advantage of affording a 
phosphinophosphonate bearing an hydroxymethylene that can be subsequently functionalized. However, no functionalization of such a reagent with a glycosyl derivative has been reported, yet and preliminary assays were not successful in our hands. In a more straightforward manner, a $H$-(phosphonyl)phosphonate reagent [31] has been condensed onto an aldehyde in smooth conditions (Figure 4D) but its preparation requires several steps and this reagent displays limited stability. In summary, the synthesis of the targeted compounds represents a major challenge.

A<smiles>[R]CP(=O)([18O])C[R]</smiles>

B<smiles>CCOP(OCC)c1ccccc1</smiles><smiles>CCOP(=O)(CCl)OCC</smiles>

C<smiles>CCCOP(=O)(C[Al]C1CCCCC1)OP(C)(=O)OC(C)C</smiles>

D<smiles>[R]C=O</smiles>
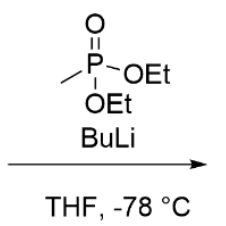<smiles>[R]CP(=O)(CP(=O)(OCC)OCC)OCC</smiles>

Reference 28<smiles>C1CCC[Te]CC1</smiles><smiles>CCOP(=O)(CP(=O)(OCC)P(=O)(OCC)c1ccccc1)OCC</smiles>

Reference 29<smiles>CCOP(=O)(CO)OC#[13C]</smiles><smiles>CCOP(=O)(CO)CP(=O)(OCC)OP(=O)(CC)OC(C)C</smiles>

Reference 30

\section{2. $\mathrm{AcOH}$}
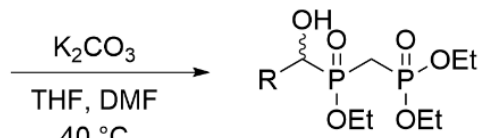

Reference 31

Figure 4. Phosphinophosphinate synthesis strategies already described on non-glycosidic molecules.

(A) Nucleophilic substitution of the chlorine atom of a chlorophosphinate by a lithio phosphonate.

(B) Michaelis-Arbuzov reaction. (C) Phospha-Claisen condensation. (D) Condensation of a $H$-(phosphonyl)phosphonate reagent onto an aldehyde.

We focused on developing a convergent strategy towards these compounds that relies on the retrosynthetic analysis outlined in Figure 5, allowing us to introduce simultaneously both phosphorus atoms in a single step according to a straightforward route. Access to the targeted phosphino-phosphonate would involve the addition of the anion of methyl-(phosphino)-phosphonate (A) onto the lactones (B) derived from commercially available $\alpha$-methyl mannoside or glucoside and $\alpha, \beta$-GlcNAc, respectively (Figure 5) leading to the corresponding lactols (C). Dehydration followed by sequential hydrogenation of the resulting double bond and deprotections would afford the targeted compounds. 

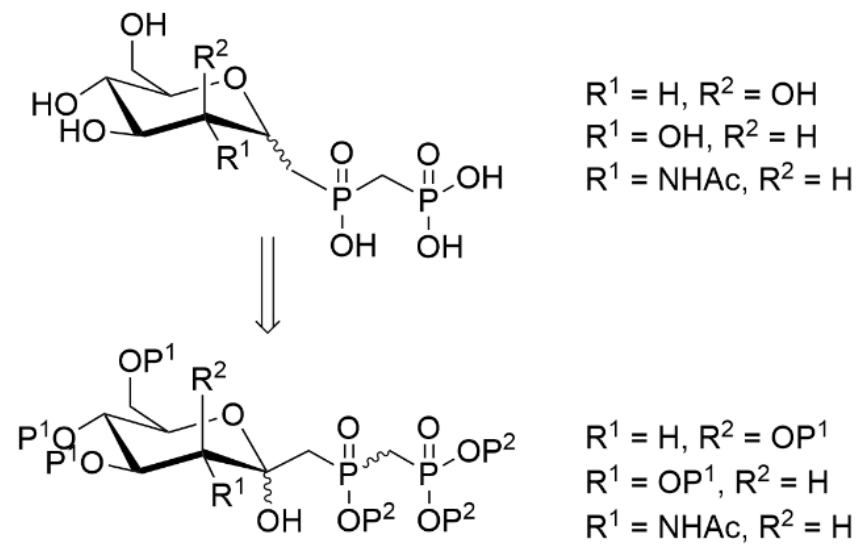

(C)

$$
\begin{aligned}
& R^{1}=O^{1}, R^{2}=H \\
& R^{1}=N H A c, R^{2}=H
\end{aligned}
$$$$
=\mathrm{H}, \mathrm{R}^{2}=\mathrm{OP}^{1}
$$

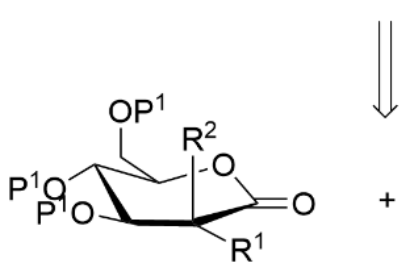

(B)

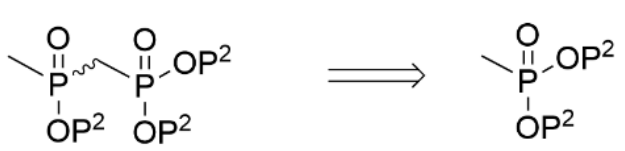

(A)

Figure 5. Retrosynthetic analysis.

\section{Results and Discussion}

\subsection{Chemistry}

We first turned our attention to the preparation of the $\delta$-hexonolactones $\mathbf{1 1 - 1 3}$ which is outlined in Scheme 1 . We aimed to develop a similar and simple pathway to access these three $\delta$-hexonolactones. The synthesis of the $\delta$-hexalactone 13 using the proposed pathway has not been reported previously. The perbenzylation of commercially available $\alpha$-methyl mannoside $\mathbf{1}$ or glucoside $\mathbf{2}$ was carried out in DMF in the presence of sodium hydride and benzyl bromide in excess to afford the protected derivatives 3 and 4 in excellent yield. Similarly, perbenzylation of a commercial $\alpha, \beta-$ GlcNAc mixture led to the benzyl acetal of perbenzylated GlcNAc 5. Deprotection at the anomeric position of compounds 3 and 4 was efficiently achieved by heating at $100{ }^{\circ} \mathrm{C}$ in the presence of hydrochloric acid in a $80 \%$ aqueous acetic acid solution giving the hemiketal 6 and 7 . It has to be noted that the deprotection at the anomeric position of compound 3 performed in the same acidic conditions was accompanied by partial deprotection of the acetamide into the corresponding amine, giving the hemiketal 8. Nevertheless, a two-step sequence involving the acetylation of compound 8 by acetic anhydride in the presence of triethylamine and dimethylaminopyridine leading to the intermediate acetamide 9 (65\% yield over two steps from 5), followed by methanolysis at the anomeric position (77\% yield) furnished the expected hemiketal 10. Oxidation at the anomeric position of lactols 6, 7 and $\mathbf{1 0}$ was efficiently carried out by molecular iodine [32] in the presence of potassium carbonate in dichloromethane to give the lactones 11 [33], 12 [34] and 13 [35], respectively.

We next turned to the preparation of diethyl ethoxy(methyl)phosphinylmethyl phosphonate 14 (Scheme 2) that was obtained in 91\% yield by autocondensation of commercially available diethyl methyl phosphonate in the presence of butyl lithium in THF [36]. 


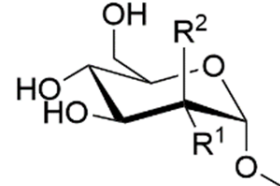

$1 \mathrm{R}^{1}=\mathrm{H}, \mathrm{R}^{2}=\mathrm{OH}$

$2 \mathrm{R}^{1}=\mathrm{OH}, \mathrm{R}^{2}=\mathrm{H}$

a)

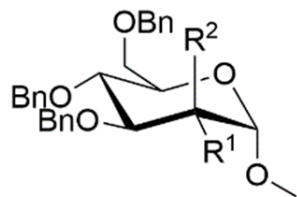

$3 \mathrm{R}^{1}=\mathrm{H}, \mathrm{R}^{2}=\mathrm{OBn}$

$4 \mathrm{R}^{1}=\mathrm{OBn}, \mathrm{R}^{2}=\mathrm{H}$

b)

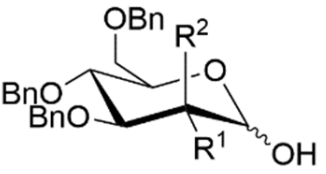

$6 \mathrm{R}^{1}=\mathrm{H}, \mathrm{R}^{2}=\mathrm{OBn}$

$7 \mathrm{R}^{1}=\mathrm{OBn}, \mathrm{R}^{2}=\mathrm{H}$

e)

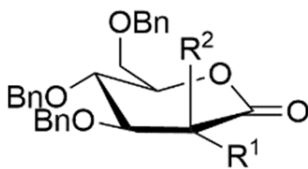

$11 R^{1}=H, R^{2}=O B n$

$12 \mathrm{R}^{1}=\mathrm{OBn}, \mathrm{R}^{2}=\mathrm{H}$

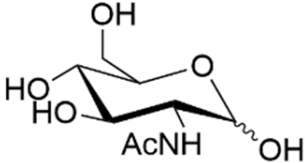

GIcNAC

a)

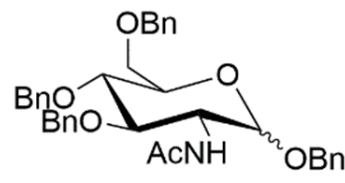

5

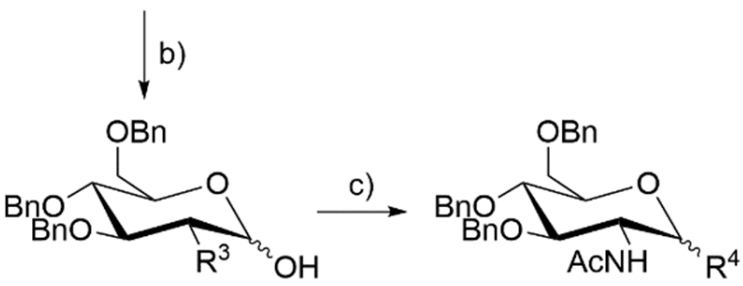

$8 \mathrm{R}^{3}=\mathrm{NHAc}$ or $\mathrm{NH}_{2}$

Scheme 1. Reagents and Conditions: (a) BnBr, NaH, DMF, $0{ }^{\circ} \mathrm{C}$ to RT, 3 days, $84 \%$ for 3, $92 \%$ for 4 , or $16 \mathrm{~h}, 67 \%$ for 5. (b) $\mathrm{HCl}, \mathrm{CH}_{3} \mathrm{COOH}, \mathrm{H}_{2} \mathrm{O}, 100{ }^{\circ} \mathrm{C}, 3 \mathrm{~h}, 58 \%$ for 6 and $58 \%$ for 7 . (c) $\mathrm{Ac}_{2} \mathrm{O}, \mathrm{Et}_{3} \mathrm{~N}$, DMAP, $\mathrm{CH}_{2} \mathrm{Cl}_{2}, 0{ }^{\circ} \mathrm{C}$ to RT, $66 \%$ from 5 over two steps. (d) $\mathrm{MeONa}, \mathrm{MeOH}, 1 \mathrm{~h}, \mathrm{RT}, 77 \%$. (e) $\mathrm{I}_{2}, \mathrm{~K}_{2} \mathrm{CO}_{3}$, $\mathrm{CH}_{2} \mathrm{Cl}_{2}, \mathrm{RT}, 16 \mathrm{~h}, 73 \%$ for $\mathbf{1 1}, 67 \%$ for $\mathbf{1 2}, 36 \%$ for 13 .<smiles>CCOP(C)(=O)OCC</smiles>

a)<smiles>CCOP(C)(=O)CP(C)(=O)OCC</smiles>

14

Scheme 2. Reagents and Conditions: (a) $n \mathrm{BuLi}, \mathrm{THF},-78^{\circ} \mathrm{C}$ to $\mathrm{RT}, 3 \mathrm{~h}, 91 \%$.

The synthesis of the targeted glycophosphinophosphonates is outlined in Scheme 3. First, the dianion of the methylphosphinyl phosphinate $\mathbf{1 4}$ was generated by the addition of a strong base, in THF, prior to the addition of the lactones 11-13. Different bases and temperature conditions were tested to efficiently perform this reaction. They notably include the sequential use of $\mathrm{NaH}$ at room temperature to remove the most acidic proton of the methylene between phosphorus atoms, followed by $n \mathrm{BuLi}$ addition at $-78^{\circ} \mathrm{C}$ [37] to generate the second anion from the methyl group or the single use of $n \mathrm{BuLi}$ at $-78^{\circ} \mathrm{C}$. These latter conditions revealed to be the most efficient. The addition of the lactones 11-13 onto 
the resulting dianion, at $-78^{\circ} \mathrm{C}$, afforded the corresponding 1-C-phosphinyl phosphonates lactols 15-17 in moderate yields [38]. Compounds 15 and 16 were present as complex mixtures of diastereoisomers resulting from two elements of chirality, $\alpha$ and $\beta$ anomers and a stereogenic phosphorus atom. For compound 17, in addition to the same diastereoisomers, rotamers, resulting from the NHAc moiety, are also observed. Then, the $\beta$-elimination of a water molecule was carried out by treatment with trifluoroactetic anhydride in the presence of pyridine in dichloromethane [39] to afford the substituted exo-glycals 18-20 in good yields and as mixtures of four possible diastereoisomers due to the presence of both the double bond and the stereogenic phosphorus atom. The stereoselective reduction of the double bond by hydrogenation [40-43] in the presence of $10 \%$ palladium on charcoal in EtOH was accompanied by benzyl ether hydrogenolysis to give the deprotected glycosyl phosphinophosphonate 21-23. These latter compounds show a single $\mathrm{R}$ configuration for the stereogenic centre $\mathrm{C} 2$ with characteristic coupling constants $\left(J_{2,3}=9.0 \mathrm{~Hz}\right.$ for 22 and $9.5 \mathrm{~Hz}$ for 26 resulting from 23) and are present as mixtures of two diastereoisomers (stereogenic phosphorus atom). For compound 21 , the $\mathrm{R}$ configuration for the stereogenic centre C2 was confirmed by NOESY experiments after acetylation. Peracetylation of compounds 21-23 by acetic anhydride in pyridine was then performed to avoid side reactions during phosphinophosphonate deprotection and afforded the corresponding compounds 24-26 in high yield. The $R$ configuration for the stereogenic centre $C 2$ of compounds 24 and 25 could be unambiguously assigned thanks to the observed correlation between $\mathrm{H}_{2}$ and $\mathrm{H}_{4}$ and $\mathrm{H}_{2}$ and $\mathrm{H}_{6}$ as demonstrated by the superimposition of COSY and NOESY spectra (see Supplementary Materials S22 and S24). Finally, the targeted compounds 27-29 were obtained according to a two-step sequence involving the deprotection of the phosphinophosphonate moiety by trimethylsilyl bromide in dichloromethane followed by saponification of the acetate protecting groups to afford the corresponding targeted compounds as single stereoisomers.

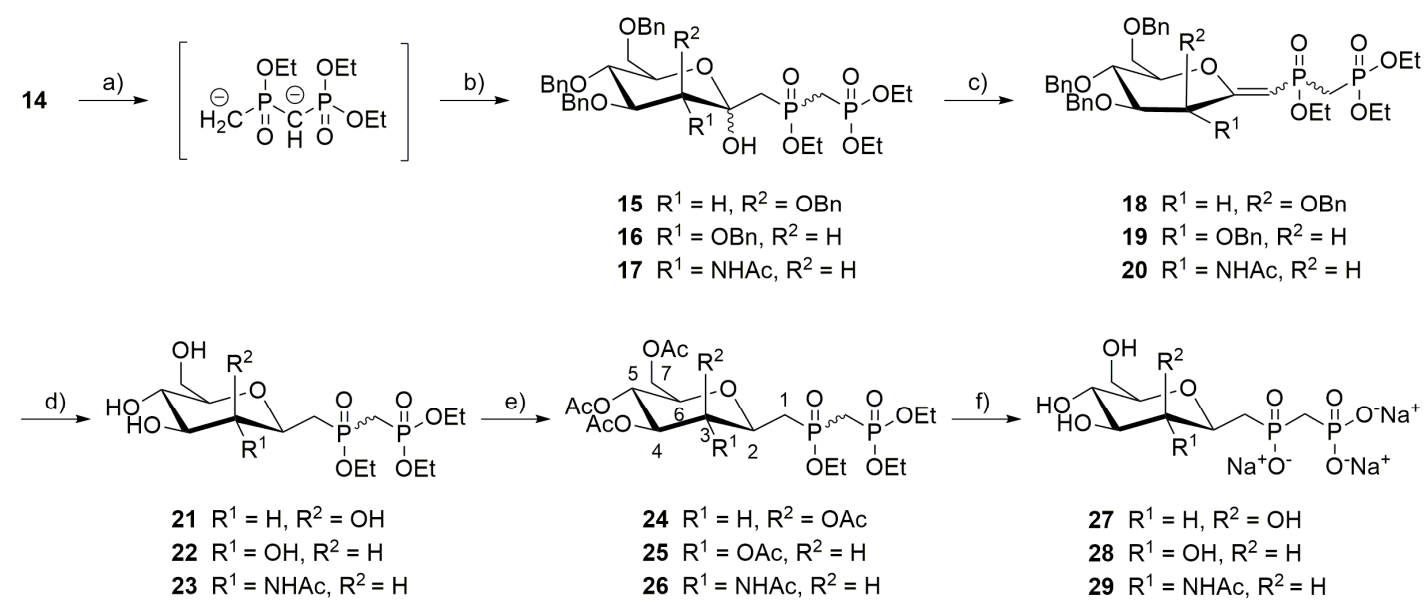

Scheme 3. Reagents and conditions: (a) $n \mathrm{BuLi}, \mathrm{THF},-78^{\circ} \mathrm{C}, 1 \mathrm{~h}$. (b) $\mathbf{1 1}, \mathbf{1 2}$ or $\mathbf{1 3}, \mathrm{THF},-78{ }^{\circ} \mathrm{C}, 2 \mathrm{~h}, 58 \%$ for $15,58 \%$ for $16,25 \%$ for 17. (c) $\left(\mathrm{CF}_{3} \mathrm{CO}\right)_{2} \mathrm{O}$, pyridine, $\mathrm{CH}_{2} \mathrm{Cl}_{2}, 0{ }^{\circ} \mathrm{C}, 3 \mathrm{~h}, 80 \%$ for $18,70 \%$ for $19,63 \%$ for 20. (d) $\mathrm{H}_{2}, \mathrm{Pd} / \mathrm{C} 10 \%, \mathrm{EtOH}, \mathrm{RT}, 16 \mathrm{~h}$. (e) $\mathrm{Ac}_{2} \mathrm{O}$, pyridine, $80 \%$ for $\mathbf{2 4}, 96 \%$ for $\mathbf{2 5}, 73 \%$ for 26 . (f) $\mathrm{i}$. TMSBr, $\mathrm{CH}_{2} \mathrm{Cl}_{2}, 0{ }^{\circ} \mathrm{C}$ to RT, 16 h; ii. $\mathrm{NaOH}, \mathrm{H}_{2} \mathrm{O}, \mathrm{RT}, 16 \mathrm{~h}, 95 \%$ for $27,92 \%$ for $28,85 \%$ for 29 .

\subsection{Biological Studies}

With these tools in hand, we next turned to their biological evaluation. Compounds 27, 28, and 29 were evaluated for their ability to inhibit di and monophosphatase activities. Dolichol linked oligosaccharide diphosphatase was used as an example of diphosphatase because we have been studying its activity for a long time [44]. Alkaline phosphatase was chosen as a representative example of monophosphatase.

Oligosaccharyl diphospho dolichol diphosphatase (DLODP) cleaves between the two phosphate residues of DLO that are required for protein $N$-glycosylation in the lumen of the endoplasmic reticulum (ER) [44]. The physiological function of this activity is not known, but oligosaccharylphosphates 
(OSP), which are products of DLODP action on DLO, are seen in increased quantities in cells derived from patients with congenital disorders of glycosylation (CDG) where truncated DLO accumulate. The proteins and genes responsible for this activity have not been identified and at present there are no known selective DLODP inhibitors. Little is known about the biochemistry of the DLODP activity but in vitro experiments using rat liver microsomes indicate that cleavage of both radioactive DLO $\left(\left[{ }^{3} \mathrm{H}\right] \mathrm{Man}_{9-5} \mathrm{GlcNAc}_{2}\right.$-PP-dolichol, [44]) and fluorescent bacterial lipid II (GlcNAc-MurNAc(dansyl-pentapeptide)-PP-undecaprenol, [45] requires detergent and $\mathrm{Co}^{2+}$. Compounds 27, 28, and 29 were tested at a concentration of $1 \mathrm{mM}$ in the standard DLODP assay using detergent-solubilized rat liver microsomes in the presence of $40 \mathrm{nM}\left[{ }^{3} \mathrm{H}\right] \mathrm{Man}_{5} \mathrm{GlcNAc}_{2}$-PP-dolichol substrate (Figure 6A). The reaction is followed by the liberation of OSP $\left(\left[{ }^{3} \mathrm{H}\right] \mathrm{Man}_{5} \mathrm{GlcNAc}_{2}-\mathrm{P}\right.$, Figure 6A). As shown in Figure 6B, at this concentration the 3 compounds had no significant effect on DLODP activity.

A

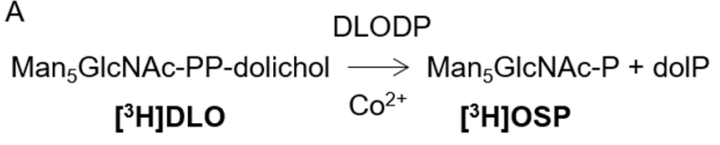

$\mathrm{B}$

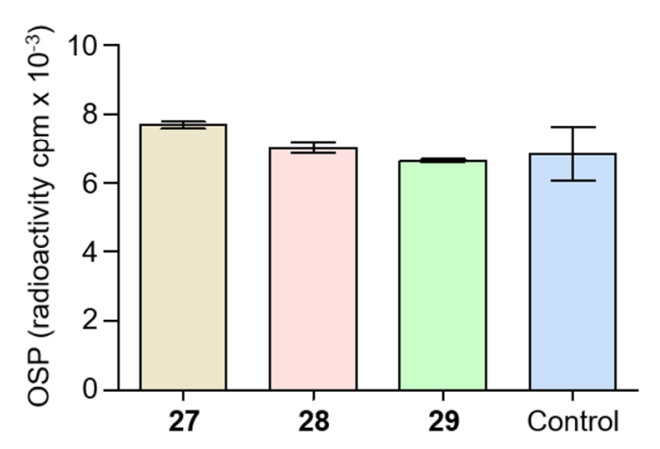

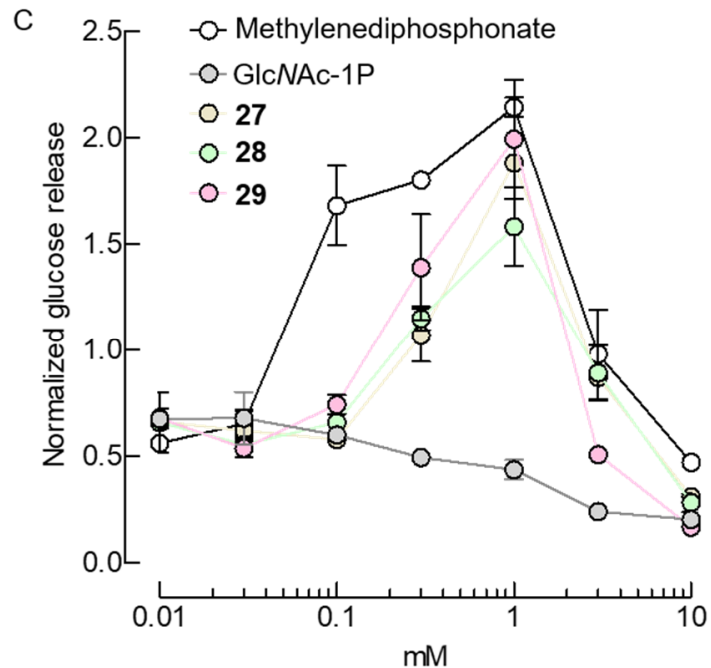

Figure 6. Evaluation of the capacity of compounds to inhibit DLO diphosphatase and alkaline phosphatase. (A) Dolichol linked oligosaccharides (DLO) are hydrolysed by oligosaccharyl diphosphodolichol diphosphatase (DLODP) to yield oligosaccharyl phosphates (OSP) and dolichyl phosphate (dolP). (B) DLODP activity was measured, as detailed in Methods, in the presence of $1 \mathrm{mM} \mathrm{27}, 28$, and 29 ( $n=2$, mean +/S.D.). (C) Alkaline phosphatase was assayed by quantitating the dephosphorylation of $\left[{ }^{3} \mathrm{H}\right] \mathrm{Glc}-1 \mathrm{P}$ as described in Methods. Compounds 27, 28, and 29 (as well as sodium methylenediphosphonate and GlcNAc-1-phosphate (GlcNAc-1P) were included in incubation mixtures at the indicated concentrations. Alkaline phosphatase activity is expressed as a fraction of that observed in control incubations conducted in the absence of added compounds ( $n=2$, mean $+/$ S.D.).

Next, we investigated the effects of compounds $\mathbf{2 7}, \mathbf{2 8}$, and $\mathbf{2 9}$ on the ability of alkaline phosphatase to dephosphorylate $\left[{ }^{3} \mathrm{H}\right] \mathrm{Glc}-1 \mathrm{P}$. Data presented in Figure $6 \mathrm{C}$ indicate complex concentration-dependent effects of these molecules on AP activity towards [ $\left.{ }^{3} \mathrm{H}\right] \mathrm{Glc}-1 \mathrm{P}$. In fact, all 3 compounds behaved similarly, and at concentrations below $0.05 \mathrm{mM}$ weakly inhibited dephosphorylation of the substrate whereas between 0.1 and $1.0 \mathrm{mM}$ they provoked 1.5-2-fold increases in the reaction. At higher concentrations, inhibition of the reaction occurred. In order to understand the origin of this complex behavior, we tested the effects of sodium methylene diphosphonate on AP-mediated dephosphorylation of $\left[{ }^{3} \mathrm{H}\right] \mathrm{Glc}-1 \mathrm{P}$. Although the potentiation phase of the dose response curve seems to occur at lower concentrations and reach a higher maximum, the data show that this compound has very similar effects to those of 27 , 28, and 29 (Figure 6C). Finally, the effects of GlcNAc-1-phosphate (GlcNAc-1P) were tested in the assay and as shown in Figure 6C this compound, in contrast to 27, 28, and 29, did not reveal an activatory capacity over the same concentration range. 


\section{Materials and Methods}

\subsection{Chemical Synthesis}

MS and/or analytical data were obtained using chromatographically homogeneous samples.

${ }^{1} \mathrm{H}$ NMR (500 MHz), ${ }^{13} \mathrm{C}$ NMR (126 MHz), and ${ }^{31} \mathrm{P}(202 \mathrm{MHz})$ spectra were recorded a Bruker Avance or Avance II (Bruker BioSpin, Wissembourg, France) in the given solvents unless otherwise indicated. Chemical shifts $(\delta)$ are reported in ppm and coupling constants are given in Hz. Low resolution mass spectra (LRMS) were recorded with an ion trap mass analyzer (LCQ Advantage, ThermoFisher, Bremen, Germany) under electrospray ionization (ESI) in positive and negative ionization mode detection. High resolution mass sprectra (HRMS) were recorded with an Orbitrap mass analyzer (Exactive, ThermoFisher, Bremen, Germany) under electrospray ionization (ESI) in positive or negative ionization mode detection, atmospheric pressure chemical ionization. All reactions were carried out under an argon atmosphere, and were monitored by thin-layer chromatography with Merck 60F-254 precoated silica (0.2 mm, Merck, Darmstadt, Germany) on glass. Flash chromatography was performed with Merck Kieselgel 60 (200-500 $\mu \mathrm{m}$, VWR, Leuven, Belgium); the solvent systems were given $v / v$.

\subsubsection{Diethyl \{[ethoxy(methyl)phosphoryl]methyl\}phosphonate $\mathbf{1 4}$}

A solution of diethyl methylphosphonate $(7.6 \mathrm{~g}, 49.6 \mathrm{mmol})$ in anhydrous THF $(15 \mathrm{~mL})$ under argon atmosphere was cooled by a dry ice/acetone bath. To this solution was added dropwise a solution of $n$-butyllithium (1.6 M in hexanes, $32 \mathrm{~mL}, 51.2 \mathrm{mmol}$ ) during $30 \mathrm{~min}$. Then the reaction mixture was allowed to warm to room temperature. After $2 \mathrm{~h}$ of stirring, the reaction was quenched by addition of an aqueous solution of hydrochloric acid (3N, $25 \mathrm{~mL})$. The reaction mixture was extracted two times with dichloromethane $(2 \times 75 \mathrm{~mL})$. The organic layers were combined, dried over anhydrous sodium sulfate, filtered, and concentrated under vacuo to obtain the crude product $(5.74 \mathrm{~g}, 22.2 \mathrm{mmol}, 89 \%)$ as a colorless oil. ${ }^{1} \mathrm{H}$ NMR $\left(500 \mathrm{MHz}, \mathrm{CDCl}_{3}\right) \delta 4.22-3.99\left(\mathrm{~m}, 6 \mathrm{H}, 3 \times \mathrm{OCH}_{2} \mathrm{CH}_{3}\right), 2.38(\mathrm{dd}, J=20.7,16.9 \mathrm{~Hz}$, $\left.2 \mathrm{H}, \mathrm{PCH}_{2} \mathrm{P}\right), 1.66\left(\mathrm{~d}, J=15.0 \mathrm{~Hz}, 3 \mathrm{H}, \mathrm{CH}_{3} \mathrm{P}\right), 1.32\left(\mathrm{t}, J=7.1 \mathrm{~Hz}, 6 \mathrm{H}, 2 \times \mathrm{OCH}_{2} \mathrm{CH}_{3}\right), 1.31(\mathrm{t}, J=7.1 \mathrm{~Hz}$, $\left.3 \mathrm{H}, \mathrm{OCH}_{2} \mathrm{CH}_{3}\right) ;{ }^{13} \mathrm{C} \mathrm{NMR}\left(126 \mathrm{MHz} \mathrm{CDCl}_{3}\right) \delta 62.6\left(\mathrm{~d}, J=6.4 \mathrm{~Hz}, \mathrm{OCH}_{2} \mathrm{CH}_{3}\right), 62.5(\mathrm{~d}, J=6.5 \mathrm{~Hz}$, $\left.\mathrm{OCH}_{2} \mathrm{CH}_{3}\right), 60.8\left(\mathrm{~d}, \mathrm{~J}=6.4 \mathrm{~Hz}, \mathrm{OCH}_{2} \mathrm{CH}_{3}\right), 29.0\left(\mathrm{dd}, J=134.5,81.2 \mathrm{~Hz}, \mathrm{PCH}_{2} \mathrm{P}\right), 16.6(\mathrm{~d}, J=6.4 \mathrm{~Hz}$, $\left.\mathrm{OCH}_{2} \mathrm{CH}_{3}\right), 16.4\left(\mathrm{~d}, \mathrm{~J}=6.3 \mathrm{~Hz}, \mathrm{OCH}_{2} \mathrm{CH}_{3}\right), 16.3\left(\mathrm{~d}, J=6.3 \mathrm{~Hz}, \mathrm{OCH}_{2} \mathrm{CH}_{3}\right), 15.8\left(\mathrm{~d}, J=100.7 \mathrm{~Hz}, \mathrm{CH}_{3} \mathrm{P}\right)$; ${ }^{31} \mathrm{P}$ NMR $\left(202 \mathrm{MHz}_{\mathrm{CDCl}}\right) \delta 44.5\left(\mathrm{~d}, J=3.4 \mathrm{~Hz}, \mathrm{CH}_{3} \mathrm{PCH}_{2} \mathrm{P}\right), 19.9\left(\mathrm{~d}, J=3.4 \mathrm{~Hz}, \mathrm{CH}_{3} \mathrm{PCH}_{2} P\right)$; ESI-MS: Calcd for $\mathrm{C}_{8} \mathrm{H}_{20} \mathrm{NaO}_{5} \mathrm{P}_{2}: 281.1[\mathrm{M}+\mathrm{Na}]^{+}$Found: 281.1; ESI-HRMS: Calcd for $\mathrm{C}_{8} \mathrm{H}_{21} \mathrm{O}_{5} \mathrm{P}_{2}: 259.0864$ $[\mathrm{M}+\mathrm{H}]^{+}$Found: 259.0865 .

\subsubsection{3,4,5,7-Tetra-O-benzyl-1-deoxy-1-\{[(diethoxyphosphoryl)methyl](ethoxy)phosphoryl $\}-\alpha$-D-} manno-2-heptulopyranose 15

A solution of diethyl \{[ethoxy(methyl)phosphoryl]methyl $\}$ phosphonate $14(1.725 \mathrm{~g}, 6.68 \mathrm{mmol})$ in anhydrous THF ( $9 \mathrm{~mL})$ under argon atmosphere was cooled by a dry ice/acetone bath. To this solution was added dropwise a solution of $n$-butyllithium (1.6 M in hexanes, $7 \mathrm{~mL}, 11.2 \mathrm{mmol})$ during $20 \mathrm{~min}$. After stirring during two hours at $-78^{\circ} \mathrm{C}$, a solution of 2,3,4,5-tetra-O-benzyl-mannono-1,5-lactone 11 $(1.2 \mathrm{~g}, 2.23 \mathrm{mmol})$ in anhydrous THF $(6 \mathrm{~mL})$ was added dropwise to the reaction mixture. After stirring during two hours at $-78^{\circ} \mathrm{C}$, the reaction was quenched by addition of a solution of acetic acid in THF $(33 \% v / v$ in THF, $6 \mathrm{~mL})$. The reaction mixture was diluted with $30 \mathrm{~mL}$ of ethyl acetate and $45 \mathrm{~mL}$ of water. To this mixture was added $12 \mathrm{~g}$ of sodium chloride. The organic layer was collected and the aqueous layer was extracted with $45 \mathrm{~mL}$ of ethyl acetate. The organic layers were combined, dried over anhydrous sodium sulfate, filtered, and concentrated. Column chromatography (silica gel $125 \mathrm{~mL}$, EtOAc then $\mathrm{EtOAc} / \mathrm{EtOH}, 98 / 2, v / v)$ of the residue afforded the product 15 as a mixture of stereoisomers (1.04 g, $1.30 \mathrm{mmol}, 58 \%)$ as a colorless oil. ${ }^{1} \mathrm{H} \mathrm{NMR}\left(500 \mathrm{MHz}, \mathrm{CDCl}_{3}\right) \delta 7.47-7.10(\mathrm{~m}, 20 \mathrm{H}, \mathrm{H}$ aromatic), 5.01-4.42 (m, 8H, $\left.4 \times \mathrm{OCH}_{2} \mathrm{Ph}\right), 4.25-3.99$ (m, 8H, H-4, $\left.3 \times \mathrm{POCH}_{2} \mathrm{CH}_{3}, \mathrm{H}-6\right), 3.94,3.91(2 \mathrm{t}, \mathrm{J}=9.7 \mathrm{~Hz}$, $1 \mathrm{H}, \mathrm{H}-5), 3.77,3.76(2 \mathrm{~d}, J=2.7 \mathrm{~Hz}, 1 \mathrm{H}, \mathrm{H}-3), 3.74-3.67$ (m, 1H, H-7), 3.67, 3.65 (2dd, J = 11.7, $2.2 \mathrm{~Hz}$, $\left.J=10.8,1.8 \mathrm{~Hz}, 1 \mathrm{H}, \mathrm{H}^{-} 7^{\prime}\right), 2.92,2.66\left(2 \mathrm{td}, J=20.5,15.8 \mathrm{~Hz}, J=20.0,15.8 \mathrm{~Hz}, 1 \mathrm{H}, \mathrm{PCH}_{2} \mathrm{P}\right), 2.85,2.74(2 \mathrm{dd}$, 
$J=15.1,11.5 \mathrm{~Hz}, J=15.3,13.6 \mathrm{~Hz}, 1 \mathrm{H}, \mathrm{H}-1), 2.52-2.30\left(\mathrm{~m}, 1 \mathrm{H}, \mathrm{PCH}_{2} \mathrm{P}\right), 2.23,1.77(2 \mathrm{dd}, J=15.3,11.5 \mathrm{~Hz}$, $\left.J=19.8,15.2 \mathrm{~Hz}, 1 \mathrm{H}, \mathrm{H}-1^{\prime}\right), 1.33-1.17\left(\mathrm{~m}, 9 \mathrm{H}, 3 \times \mathrm{POCH}_{2} \mathrm{CH}_{3}\right) ;{ }^{13} \mathrm{C} \mathrm{NMR}\left(126 \mathrm{MHz}, \mathrm{CDCl}_{3}\right) \delta 138.75$, 138.72, 138.66, 138.64, 138.59, 138.50, 138.46 ( $4 \times$ Cq aromatic), 128.56, 128.54, 128.42, 128.39, 128.3, $128.13,128.08,127.91,127.88,127.8,127.71,127.69,127.60(20 \times \mathrm{CH}$ aromatic $), 98.2,97.8(2 \mathrm{~d}, J=8.8 \mathrm{~Hz}$, $J=5.5 \mathrm{~Hz}, \mathrm{C}-2), 81.5,81.4(2 \mathrm{~d}, J=2.5 \mathrm{~Hz}, J=2.3 \mathrm{~Hz}, \mathrm{C}-4), 78.9,78.4(2 \mathrm{~d}, J=9.7 \mathrm{~Hz}, J=9.2 \mathrm{~Hz}, \mathrm{C}-3), 75.2$ $\left(\mathrm{OCH}_{2} \mathrm{Ph}\right), 75.0,74.9,74.8,73.3,73.2,73.03,72.97\left(4 \times \mathrm{OCH}_{2} \mathrm{Ph}\right), 75.1(\mathrm{C}-5), 72.4,71.9(\mathrm{C}-6), 69.8,69.7$ (C-7), 63.0, 62.8, 62.7, 62.6, 62.44, $61.39(6 \mathrm{~d}, J=6.4 \mathrm{~Hz}, J=6.3 \mathrm{~Hz}, J=6.5 \mathrm{~Hz}, J=6.6 \mathrm{~Hz}, J=6.4 \mathrm{~Hz}$, $\left.J=6.7 \mathrm{~Hz}, 3 \times \mathrm{OCH}_{2} \mathrm{CH}_{3}\right), 37.3,35.2(2 \mathrm{~d}, J=92 \mathrm{~Hz}, J=94 \mathrm{~Hz}, \mathrm{C}-1), 30.2,29.1(2 \mathrm{dd}, J=135,87 \mathrm{~Hz}$, $\left.J=134,84 \mathrm{~Hz}, \mathrm{PCH}_{2} \mathrm{P}\right), 16.58-16.29\left(\mathrm{~m}, 3 \times \mathrm{POCH}_{2} \mathrm{CH}_{3}\right) ;{ }^{31} \mathrm{P} \mathrm{NMR}\left(202 \mathrm{MHz}, \mathrm{CDCl}_{3}\right) \delta 48.3,46.7(2 \mathrm{~d}$, $\left.J=11.5 \mathrm{~Hz}, J=3.4 \mathrm{~Hz}, \mathrm{CH}_{2} P \mathrm{PCH}_{2} \mathrm{P}\right), 20.0,19.6\left(2 \mathrm{~d}, J=11.5 \mathrm{~Hz}, J=3.4 \mathrm{~Hz}, 1 \mathrm{P}, \mathrm{CH}_{2} \mathrm{PCH}_{2} P\right)$; ESI-MS: Calcd for $\mathrm{C}_{42} \mathrm{H}_{54} \mathrm{NaO}_{11} \mathrm{P}_{2}: 819.8[\mathrm{M}+\mathrm{Na}]^{+}$Found: 819.2; ESI-HRMS: Calcd for $\mathrm{C}_{42} \mathrm{H}_{54} \mathrm{NaO}_{11} \mathrm{P}_{2}$ : $819.3034[\mathrm{M}+\mathrm{Na}]^{+}$Found: 819.3035.

3.1.3. 3,4,5,7-Tetra-O-benzyl-1-deoxy-1-\{[(diethoxyphosphoryl)methyl](ethoxy)phosphoryl\} - $\alpha$-D-gluco-2- heptulopyranose 16

A solution of diethyl \{[ethoxy(methyl)phosphoryl]methyl $\}$ phosphonate $14(2.87 \mathrm{~g}, 11.1 \mathrm{mmol})$ in anhydrous THF $(15 \mathrm{~mL})$ under argon atmosphere was cooled by a dry ice/acetone bath. To this solution was added dropwise a solution of $n$-butyllithium $(1.6 \mathrm{M}$ in hexanes, $11.6 \mathrm{~mL}, 18.6 \mathrm{mmol})$ during $20 \mathrm{~min}$. After stirring during two hours at $-78^{\circ} \mathrm{C}$, a solution of 2,3,4,5-tetra-O-benzyl-glucono-1,5-lactone 12 $(2 \mathrm{~g}, 3.71 \mathrm{mmol})$ in anhydrous THF $(10 \mathrm{~mL})$ was added dropwise to the reaction mixture. After stirring during two hours at $-78^{\circ} \mathrm{C}$, the reaction was quenched by addition of a solution of acetic acid in THF $(28 \% v / v$ in THF, $7 \mathrm{~mL}$ ). The reaction mixture was diluted with $50 \mathrm{~mL}$ of ethyl acetate and $75 \mathrm{~mL}$ of water. To this mixture was added $20 \mathrm{~g}$ of sodium chloride. The organic layer was collected and the aqueous layer was extracted with $75 \mathrm{~mL}$ of ethyl acetate. The organic layers were combined, dried over anhydrous sodium sulfate, filtered, and concentrated. Column chromatography (silica gel $200 \mathrm{~mL}$, EtOAc) of the residue afforded the product 16 as a mixture of stereoisomers $(1.736 \mathrm{~g}, 2.17 \mathrm{mmol}, 58 \%)$ as a colorless oil. ${ }^{1} \mathrm{H}$ NMR $\left(500 \mathrm{MHz}, \mathrm{CDCl}_{3}\right) \delta 7.41-7.12(\mathrm{~m}, 20 \mathrm{H}, \mathrm{H}$ aromatic), 4.98, 4.96, 4.92, 4.91, $4.89,4.88,4.84,4.83,4.69,4.65,4.57,4.55,4.53,4.50,4.45,4.44(16 \mathrm{~d}, J=11.7 \mathrm{~Hz}, J=11.4 \mathrm{~Hz}, J=11.0 \mathrm{~Hz}$, $J=10.9 \mathrm{~Hz}, J=10.9 \mathrm{~Hz}, J=11.0 \mathrm{~Hz}, J=11.0 \mathrm{~Hz}, J=11.0 \mathrm{~Hz}, J=11.4 \mathrm{~Hz}, J=11.7 \mathrm{~Hz}, J=9.8 \mathrm{~Hz}$, $\left.J=9.8 \mathrm{~Hz}, J=11.8 \mathrm{~Hz}, J=11.8 \mathrm{~Hz}, J=11.8 \mathrm{~Hz}, J=11.8 \mathrm{~Hz}, 8 \mathrm{H}, 4 \times \mathrm{OCH}_{2} \mathrm{Ph}\right), 4.22-3.94(\mathrm{~m}, 8 \mathrm{H}, 3 \times$ $\left.\mathrm{OCH}_{2} \mathrm{CH}_{3}, \mathrm{H}-6, \mathrm{H}-4\right), 3.75-3.56$ (m, 3H, H-7, H-7' , H-5), 3.34, 3.27 (2d, J = 9.5 Hz, 1H, H-3), 2.92-2.02 (m, 4H, H-1, $\left.\mathrm{PCH}_{2} \mathrm{P}, \mathrm{H}-1^{\prime}\right), 1.37-1.20\left(\mathrm{~m}, 9 \mathrm{H}, 3 \times \mathrm{OCH}_{2} \mathrm{CH}_{3}\right) ;{ }^{13} \mathrm{C} \mathrm{NMR}\left(126 \mathrm{MHz}, \mathrm{CDCl}_{3}\right) \delta 138.8$, 138.7, 138.4, 138.3, 138.2, 138.1 (4 $\times$ Cq aromatic), 128.62, 128.58, 128.56, 128.53, 128.52, 128.51, 128.49, $128.04,128.03,128.00,127.94,127.92,127.90,127.87,127.86,127.82,127.80,127.76,127.70(20 \times \mathrm{CH}$ aromatic), 97.7, $97.3(2 \mathrm{~d}, J=8.4 \mathrm{~Hz}, J=6.8 \mathrm{~Hz}, \mathrm{C}-2), 83.7,83.6(2 \mathrm{~d}, J=11.1 \mathrm{~Hz}, J=10.0 \mathrm{~Hz}, \mathrm{C}-3), 83.2$ $(2 \mathrm{~d}, J=3.0 \mathrm{~Hz}, J=3.6 \mathrm{~Hz}, \mathrm{C}-4), 79.0,78.6(\mathrm{C}-5), 75.8,75.7,75.6,75.5,75.0,73.5,73.2\left(4 \times \mathrm{OCH}_{2} \mathrm{Ph}\right)$, 71.2, 70.6 (C-6), 69.4, 69.1 (C-7), 62.8, 62.7, 62.6, 62.3, 62.1, $61.4(6 \mathrm{~d}, J=6.4 \mathrm{~Hz}, J=6.4 \mathrm{~Hz}, J=6.4 \mathrm{~Hz}$, $\left.J=6.5 \mathrm{~Hz}, J=6.3 \mathrm{~Hz}, J=6.6 \mathrm{~Hz}, 3 \times \mathrm{OCH}_{2} \mathrm{CH}_{3}\right), 37.5,34.8(2 \mathrm{~d}, J=91.7 \mathrm{~Hz}, J=91.3 \mathrm{~Hz}, \mathrm{C}-1), 30.2,29.3$ $\left(2 \mathrm{dd}, J=133.8,86.3 \mathrm{~Hz}, J=134.1,85.4 \mathrm{~Hz}, \mathrm{PCH}_{2} \mathrm{P}\right), 16.6-16.3\left(\mathrm{~m}, 3 \times \mathrm{OCH}_{2} \mathrm{CH}_{3}\right) ;{ }^{31} \mathrm{P} \mathrm{NMR}(202 \mathrm{MHz}$, $\left.\mathrm{CDCl}_{3}\right) \delta 48.3,45.8\left(2 \mathrm{~d}, J=11.6 \mathrm{~Hz}, J=6.6 \mathrm{~Hz}, \mathrm{CH}_{2} P \mathrm{PCH}_{2} \mathrm{P}\right), 19.6,19.5(2 \mathrm{~d}, J=11.6 \mathrm{~Hz}, J=6.6 \mathrm{~Hz}$, $\mathrm{CH}_{2} \mathrm{PCH}_{2} \mathrm{P}$ ); ESI-MS: Calcd for $\mathrm{C}_{42} \mathrm{H}_{54} \mathrm{NaO}_{11} \mathrm{P}_{2}: 819.8$ [M + Na] ${ }^{+}$Found: 819.1; ESI-HRMS: Calcd for $\mathrm{C}_{42} \mathrm{H}_{55} \mathrm{O}_{11} \mathrm{P}_{2}: 797.3214[\mathrm{M}+\mathrm{H}]^{+}$Found: 797.3228.

3.1.4. 3-Acetamido-4,5,7-tri-O-benzyl-1,3-di-deoxy-1-\{[(diethoxyphosphoryl)methyl](ethoxy) phosphoryl\}- $\alpha$-D-gluco-2-heptulopyranose 17

A solution of diethyl \{[ethoxy(methyl)phosphoryl]methyl\}phosphonate 14 (1.845 g, $7.15 \mathrm{mmol})$ in anhydrous THF $(9 \mathrm{~mL})$ under argon atmosphere was cooled by a dry ice/acetone bath. To this solution was added dropwise a solution of $n$-butyllithium (1.6 M in hexanes, $7.6 \mathrm{~mL}, 12.25 \mathrm{mmol})$ during $20 \mathrm{~min}$. After stirring during two hours at $-78{ }^{\circ} \mathrm{C}$, a solution of 2-acetamido-3,4,5-tri-O-benzyl2-deoxy-glucono-1,5-lactone 13 (1 g, $2.04 \mathrm{mmol})$ in anhydrous THF $(6 \mathrm{~mL})$ was added dropwise to the 
reaction mixture. After stirring during two hours at $-78^{\circ} \mathrm{C}$, the reaction was quenched by addition of a solution of acetic acid in THF (11\% v/v in THF, $4.5 \mathrm{~mL})$. The reaction mixture was diluted with $45 \mathrm{~mL}$ of ethyl acetate and $45 \mathrm{~mL}$ of water. To this mixture was added $12 \mathrm{~g}$ of sodium chloride. The organic layer was collected and the aqueous layer was extracted with $45 \mathrm{~mL}$ of ethyl acetate. The organic layers were combined, dried over anhydrous sodium sulfate, filtered, and concentrated. Column chromatography (silica gel $150 \mathrm{~mL}$, EtOAc/EtOH, 95/5 to 9/1, v/v) of the residue afforded the product 17 as a mixture of stereoisomers $(387 \mathrm{mg}, 517 \mu \mathrm{mol}, 25 \%)$ as a colorless oil. ${ }^{1} \mathrm{H} \mathrm{NMR}\left(500 \mathrm{MHz}, \mathrm{CDCl}_{3}\right)$ $\delta$ 7.39-7.14 (m, 15H, H aromatic), 6.42, $6.35(2 \mathrm{~s}, 1 \mathrm{H}, \mathrm{C}-\mathrm{OH}), 5.58,5.44(2 \mathrm{~d}, J=10.0 \mathrm{~Hz}, J=10.1 \mathrm{~Hz}$, $\left.1 \mathrm{H}, \mathrm{NHCOCH}_{3}\right), 4.83,4.82,4.81,4.64,4.56,4.55,4.55,4.49,4.48,4.45(10 \mathrm{~d}, J=10.9 \mathrm{~Hz}, J=11.5 \mathrm{~Hz}$, $J=10.9 \mathrm{~Hz}, J=11.5 \mathrm{~Hz}, J=10.9 \mathrm{~Hz}, J=10.9 \mathrm{~Hz}, J=12.0 \mathrm{~Hz}, J=12.0 \mathrm{~Hz}, J=12.0 \mathrm{~Hz}, J=12.0 \mathrm{~Hz}$, $\left.6 \mathrm{H}, 3 \times \mathrm{OCH}_{2} \mathrm{Ph}\right), 4.21-3.98$ (m, 8H, $\left.3 \times \mathrm{OCH}_{2} \mathrm{CH}_{3}, \mathrm{H}-6, \mathrm{H}-3\right), 3.77-3.59$ (m, 4H, H-7, H-7' , H-4, H-5), 2.92-2.32 (m, 3H, PCH $\left.\mathrm{P}_{2} \mathrm{H}-1\right), 2.21,2.12\left(\mathrm{t}, \mathrm{dd}, J=14.7 \mathrm{~Hz}, J=19.7,15.3 \mathrm{~Hz}, 1 \mathrm{H}, \mathrm{H}-1^{\prime}\right), 1.88,1.86(2 \mathrm{~s}, 3 \mathrm{H}$, $\left.\mathrm{NHCOCH}_{3}\right), 1.38-1.23\left(\mathrm{~m}, 9 \mathrm{H}, 3 \times \mathrm{OCH}_{2} \mathrm{CH}_{3}\right) ;{ }^{13} \mathrm{C} \mathrm{NMR}\left(126 \mathrm{MHz}, \mathrm{CDCl}_{3}\right) \delta 170.3,170.1\left(\mathrm{NHCOCH}_{3}\right)$, 138.7, 138.6, 138.3, 138.21, 138.17, 138.1 (3 × Cq aromatic), 128.62, 128.57, 128.56, 128.55, 128.52, 128.2, $128.13,128.11,127.93,127.89,127.81,127.77(15 \times \mathrm{CH}$ aromatic $), 98.1,97.4(2 \mathrm{~d}, J=9.2 \mathrm{~Hz}, J=6.5 \mathrm{~Hz}$, C-2), 80.9, $80.8(2 \mathrm{~d}, J=3.7 \mathrm{~Hz}, J=3.0 \mathrm{~Hz}, \mathrm{C}-4), 78.7,75.2,75.0,73.4,73.3\left(3 \times \mathrm{OCH}_{2} \mathrm{Ph}\right), 71.3,71.0(\mathrm{C}-6)$, $69.3,69.1$ (C-7), 63.0, 62.8, 62.6, 62.4, $61.7(5 \mathrm{~d}, J=6.3 \mathrm{~Hz}, J=6.5 \mathrm{~Hz}, J=6.5 \mathrm{~Hz}, J=6.3 \mathrm{~Hz}, J=6.3 \mathrm{~Hz}$, $\left.\mathrm{OCH}_{2} \mathrm{CH}_{3}\right), 57.4,57.2(2 \mathrm{~d}, J=11.2 \mathrm{~Hz}, J=11.7 \mathrm{~Hz}, \mathrm{C}-3), 37.0,35.6(2 \mathrm{~d}, J=90.3 \mathrm{~Hz}, J=94.0 \mathrm{~Hz}, \mathrm{C}-1)$, 30.1, $29.2\left(2 \mathrm{dd}, J=134.7,88.3 \mathrm{~Hz}, J=133.7,83.4 \mathrm{~Hz}, \mathrm{PCH}_{2} \mathrm{P}\right), 23.63,23.61\left(\mathrm{NHCOCH}_{3}\right), 16.6-16.3(\mathrm{~m}, 3$ $\left.\times \mathrm{OCH}_{2} \mathrm{CH}_{3}\right) ;{ }^{31} \mathrm{P} \mathrm{NMR}\left(2 \mathrm{MHz}, \mathrm{CDCl}_{3}\right) \delta 48.2,46.5\left(2 \mathrm{~d}, J=12.5 \mathrm{~Hz}, J=1.8 \mathrm{~Hz}, \mathrm{CH}_{2} P \mathrm{CH}_{2} \mathrm{P}\right), 19.4$, $19.1\left(2 \mathrm{~d}, J=12.5 \mathrm{~Hz}, J=1.8 \mathrm{~Hz}, \mathrm{CH}_{2} \mathrm{PCH}_{2} \mathrm{P}\right)$; ESI-MS: Calcd for $\mathrm{C}_{37} \mathrm{H}_{51} \mathrm{NNaO}_{11} \mathrm{P}_{2}: 770.3[\mathrm{M}+\mathrm{Na}]^{+}$ Found: 770.1; ESI-HRMS: Calcd for $\mathrm{C}_{37} \mathrm{H}_{52} \mathrm{NO}_{11} \mathrm{P}_{2}$ : $748.3010[\mathrm{M}+\mathrm{H}]^{+}$Found: 748.3036.

3.1.5. 2,6-Anhydro-3,4,5,7-tetra-O-benzyl-1-deoxy-1-\{[(diethoxyphosphoryl)methyl](ethoxy) phosphoryl\}-D-manno-hept-1-enitol 18

To a solution of heptulopyranose $15(0.9 \mathrm{~g}, 1.13 \mathrm{mmol})$ in anhydrous dichlormethane $(35 \mathrm{~mL})$ under argon atmopshere and cooled by an ice bath, was added dropwise pyridine ( $3.5 \mathrm{~mL}, 43.3 \mathrm{mmol})$ then trifluoroacetic anhydride $(0.66 \mathrm{~mL}, 4.75 \mathrm{mmol})$. After stirring three hours at $0{ }^{\circ} \mathrm{C}$, the reaction is quenched by the addition of an aqueous saturated solution of sodium bicarbonate $(70 \mathrm{~mL})$. The organic layer was collected and the aqueous layer was extracted two times with $50 \mathrm{~mL}$ of dichloromethane. The organic layers were combined, washed with $70 \mathrm{~mL}$ of an aqueous solution of hydrochloric acid $1 \mathrm{~N}$, then $70 \mathrm{~mL}$ of brine, dried over anhydrous sodium sulfate, filtered, and concentrated. Column chromatography (silica gel $100 \mathrm{~mL}, \mathrm{EtOAc} / \mathrm{EtOH}, 98 / 2$ to 9/1, v/v) of the residue afforded three fractions of diastereoisomers 18 isolated as colorless oils (94 mg, $120 \mu \mathrm{mol}, 10 \% ; 77 \mathrm{mg}, 99 \mu \mathrm{mol}, 9 \% ; 537 \mathrm{mg}$, $689 \mu \mathrm{mol}, 61 \%)$. The physicochemical characteristics of the major fraction are reported. ${ }^{1} \mathrm{H} \mathrm{NMR}$ $\left(500 \mathrm{MHz} \mathrm{CDCl}_{3}\right) \delta 7.43-7.16(\mathrm{~m}, 20 \mathrm{H}, \mathrm{H}$ aromatic), $5.20(2 \mathrm{~d}, J=11.9 \mathrm{~Hz}, J=9.7 \mathrm{~Hz}, 1 \mathrm{H}, \mathrm{H}-1), 4.79-4.45$ $\left(\mathrm{m}, 8 \mathrm{H}, 4 \times \mathrm{OCH}_{2} \mathrm{Ph}\right), 4.21-4.06(\mathrm{~m}, 1 \mathrm{H}, \mathrm{H}-3), 4.16-3.91\left(\mathrm{~m}, 9 \mathrm{H}, 3 \times \mathrm{POCH}_{2} \mathrm{CH}_{3}, \mathrm{H}-2, \mathrm{H}-5, \mathrm{H}-6\right)$, 3.85-3.66 (m, 3H, H-4, H-7, H-7'), 2.85-2.52 (m, 2H, PCH $\left.{ }_{2} \mathrm{P}\right), 1.30-1.23\left(\mathrm{~m}, 9 \mathrm{H}, 3 \times \mathrm{POCH}_{2} \mathrm{CH}_{3}\right) ;{ }^{13} \mathrm{C}$ NMR $\left(126 \mathrm{MHz} \mathrm{CDCl}_{3}\right) \delta 164.6,164.0$ (s, d, J = 1.3 Hz, C-2), 138.2, 138.1, 138.0, 137.95, 137.91, 137.8, $137.42,137.36$ ( $4 \times$ Cq aromatic) $, 128.64,128.60,128.55,128.54,128.50,128.2,128.1,128.03,128.00,127.94$, 127.92, 127.82, $127.78(20 \times \mathrm{CH}$ aromatic), 100.5, $97.7(2 \mathrm{~d}, J=136.4 \mathrm{~Hz}, J=137.5 \mathrm{~Hz}, \mathrm{C}-1), 79.7,78.9$ (C-6), 78.8, 77.9 (C-4), 75.2, $74.6(2 \mathrm{~d}, J=12.0 \mathrm{~Hz}, J=12.2 \mathrm{~Hz}, \mathrm{C}-3), 74.9,74.4$ (C-5), 74.2, 73.52, 73.48, 73.4, 72.5, 72.1, 71.6, $71.0\left(4 \times \mathrm{OCH}_{2} \mathrm{Ph}\right), 69.5(\mathrm{C}-7), 62.5,62.44,62.39,62.3,61.00,60.98(6 \mathrm{~d}, \mathrm{~J}=6.3 \mathrm{~Hz}$, $\left.J=6.3 \mathrm{~Hz}, J=6.5 \mathrm{~Hz}, J=6.2 \mathrm{~Hz}, J=6.1 \mathrm{~Hz}, J=5.8 \mathrm{~Hz}, 3 \mathrm{xOCH}_{2} \mathrm{CH}_{3}\right), 29.2,28.9(2 \mathrm{dd}, J=134,92.5 \mathrm{~Hz}$, $\left.J=134,94 \mathrm{~Hz}, \mathrm{PCH}_{2} \mathrm{P}\right), 16.62,16.56,16.48,16.47,16.45(5 \mathrm{~d}, J=7.9 \mathrm{~Hz}, J=7.1 \mathrm{~Hz}, J=6.3 \mathrm{~Hz}, J=5.9 \mathrm{~Hz}$, $\left.J=6.4 \mathrm{~Hz}, \mathrm{OCH}_{2} \mathrm{CH}_{3}\right) ;{ }^{31} \mathrm{P} \mathrm{NMR}\left(202 \mathrm{MHz} \mathrm{CDCl}_{3}\right) \delta 31.1,30.4\left(2 \mathrm{~d}, J=3.6 \mathrm{~Hz}, J=7.8 \mathrm{~Hz}, \mathrm{CH}_{2} P C \mathrm{CH}_{2} \mathrm{P}\right)$, 20.5, $20.4\left(2 \mathrm{~d}, J=3.6 \mathrm{~Hz}, J=7.8 \mathrm{~Hz}, \mathrm{CH}_{2} \mathrm{PCH}_{2} \mathrm{P}\right)$; ESI-MS: Calcd for $\mathrm{C}_{42} \mathrm{H}_{52} \mathrm{NaO}_{10} \mathrm{P}_{2}: 801.8[\mathrm{M}+\mathrm{Na}]^{+}$ Found: 801.1; ESI-HRMS: Calcd for $\mathrm{C}_{42} \mathrm{H}_{53} \mathrm{O}_{10} \mathrm{P}_{2}$ : $779.3108[\mathrm{M}+\mathrm{H}]^{+}$Found: 779.3140 . 
3.1.6. 2,6-Anhydro-3,4,5,7-tetra-O-benzyl-1-deoxy-1-\{[(diethoxyphosphoryl)methyl](ethoxy) phosphoryl\}-D-gluco-hept-1-enitol 19

To a solution of heptulopyranose $16(1.1 \mathrm{~g}, 1.38 \mathrm{mmol})$ in anhydrous dichloromethane $(41 \mathrm{~mL})$ under argon atmosphere and cooled by an ice bath, was added dropwise pyridine $(4.1 \mathrm{~mL}, 50.7 \mathrm{mmol})$ then trifluoroacetic anhydride $(0.77 \mathrm{~mL}, 5.52 \mathrm{mmol})$. After stirring three hours at $0{ }^{\circ} \mathrm{C}$, the reaction is quenched by the addition of an aqueous saturated solution of sodium bicarbonate $(80 \mathrm{~mL})$. The organic layer was collected and the aqueous layer was extracted two times with $60 \mathrm{~mL}$ of dichloromethane. The organic layers were combined, washed with $80 \mathrm{~mL}$ of an aqueous solution of hydrochloric acid $1 \mathrm{~N}$, then $80 \mathrm{~mL}$ of brine, dried over anhydrous sodium sulfate, filtered, and concentrated. Column chromatography (silica gel $100 \mathrm{~mL}, \mathrm{EtOAc} / \mathrm{EtOH}, 98 / 2$ to 9/1, v/v) of the residue afforded the product 19 as a mixture of stereoisomers $(0.75 \mathrm{~g}, 0.96 \mathrm{mmol}, 70 \%)$ as a colorless oil. ${ }^{1} \mathrm{H}$ NMR $\left(500 \mathrm{MHz}, \mathrm{CDCl}_{3}\right)$

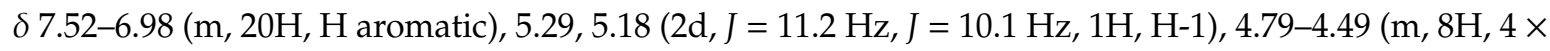
$\left.\mathrm{OCH}_{2} \mathrm{Ph}\right), 4.27-3.97\left(\mathrm{~m}, 7 \mathrm{H}, 3 \times \mathrm{OCH}_{2} \mathrm{CH}_{3}, \mathrm{H}-6\right), 3.97-3.93(\mathrm{~m}, 1 \mathrm{H}, \mathrm{H}-3), 3.84-3.76(\mathrm{~m}, 3 \mathrm{H}, \mathrm{H}-4, \mathrm{H}-5$, $\mathrm{H}-7), 3.73,3.71\left(2 \mathrm{dd}, J=12.3,4.1 \mathrm{~Hz}, J=12.2,4.0 \mathrm{~Hz}, 1 \mathrm{H}, \mathrm{H}-7^{\prime}\right), 2.93-2.47\left(\mathrm{~m}, 2 \mathrm{H}, \mathrm{PCH}_{2} \mathrm{P}\right), 1.31-1.25$ $\left(\mathrm{m}, 9 \mathrm{H}, 3 \times \mathrm{OCH}_{2} \mathrm{CH}_{3}\right) ;{ }^{13} \mathrm{C}$ NMR $\left(126 \mathrm{MHz}, \mathrm{CDCl}_{3}\right) \delta 164.36,164.2(\mathrm{~d}, \mathrm{~s}, J=1.1 \mathrm{~Hz}, \mathrm{C}-2), 138.0,137.92$, 137.86, 137.8, 137.7, 137.3, 137.2 ( $4 \times$ Cq aromatic), 128.7, 128.62, 128.56, 128.55, 128.53, 128.50, 128.47, $128.2,128.13,128.09,128.08,128.05,128.02,128.00,127.98,127.96,127.91,127.90,127.83,127.81(20 \mathrm{C}$, $\mathrm{CH}$ aromatic), 99.3, $99.0(2 \mathrm{~d}, J=136.8 \mathrm{~Hz}, J=136.9 \mathrm{~Hz}, \mathrm{C}-1), 83.6,82.9(\mathrm{C}-4), 78.7,77.9(2 \mathrm{~d}, J=11.9 \mathrm{~Hz}$, $J=12.1 \mathrm{~Hz}, \mathrm{C}-3), 78.2,77.6$ (C-6), 77.4, 77.10 (C-5), 74.3, 73.90, 73.85, 73.56, 73.51, 73.49, 72.8, 72.2 (4 × $\left.\mathrm{OCH}_{2} \mathrm{Ph}\right), 68.5,68.4(\mathrm{C}-7), 62.5,62.42,62.35,62.33,61.00,60.98(6 \mathrm{~d}, J=6.2 \mathrm{~Hz}, J=6.2 \mathrm{~Hz}, J=6.3 \mathrm{~Hz}$, $\left.J=6.2 \mathrm{~Hz}, J=6.1 \mathrm{~Hz}, J=6.1 \mathrm{~Hz}, 3 \times \mathrm{OCH}_{2} \mathrm{CH}_{3}\right), 29.1\left(\mathrm{dd}, J=134.4,93.1 \mathrm{~Hz}, \mathrm{CHPCH}_{2} \mathrm{P}\right), 16.7-16.4(\mathrm{~m}$, $\left.3 \times \mathrm{OCH}_{2} \mathrm{CH}_{3}\right) ;{ }^{31} \mathrm{P} \mathrm{NMR}\left(202 \mathrm{MHz}, \mathrm{CDCl}_{3}\right) \delta 30.8,30.6\left(2 \mathrm{~d}, J=7.7 \mathrm{~Hz}, J=6.5 \mathrm{~Hz}, \mathrm{CH}_{2} P_{C H} \mathrm{P}\right), 20.5$, $20.5\left(2 \mathrm{~d}, J=7.7 \mathrm{~Hz}, J=6.5 \mathrm{~Hz}, \mathrm{CH}_{2} \mathrm{PCH}_{2} P\right)$; ESI-MS: Calcd for $\mathrm{C}_{42} \mathrm{H}_{53} \mathrm{O}_{10} \mathrm{P}_{2}: 779.8[\mathrm{M}+\mathrm{H}]^{+}$Found: 779.0, Calcd for $\mathrm{C}_{42} \mathrm{H}_{54} \mathrm{KO}_{10} \mathrm{P}_{2}: 819.9[\mathrm{M}+2 \mathrm{H}+\mathrm{K}]^{+}$Found: 819.2; ESI-HRMS: Calcd for $\mathrm{C}_{42} \mathrm{H}_{53} \mathrm{O}_{10} \mathrm{P}_{2}$ : $779.3108[\mathrm{M}+\mathrm{H}]^{+}$Found: 779.3127.

3.1.7. 3-Acetamido-2,6-Anhydro-4,5,7-tri-O-benzyl-1,3-di-deoxy-1-\{[(diethoxyphosphoryl) methyl](ethoxy)phosphoryl\}-D-gluco-hept-1-enitol 20

To a solution of heptulopyranose $\mathbf{1 7}(340 \mathrm{mg}, 455 \mu \mathrm{mol})$ in anhydrous dichlormethane (14 mL) under argon atmosphere and cooled by an ice bath, was added dropwise pyridine $(1.4 \mathrm{~mL}, 17.3 \mathrm{mmol})$ then trifluoroacetic anhydride $(250 \mu \mathrm{L}, 1.82 \mathrm{mmol})$. After stirring two hours at $0{ }^{\circ} \mathrm{C}$, the reaction is quenched by the addition of an aqueous saturated solution of sodium bicarbonate $(26 \mathrm{~mL})$. The organic layer was collected and the aqueous layer was extracted two times with $26 \mathrm{~mL}$ of dichloromethane. The organic layers were combined, washed with $26 \mathrm{~mL}$ of an aqueous solution of hydrochloric acid $1 \mathrm{~N}$, then $26 \mathrm{~mL}$ of brine, dried over anhydrous sodium sulfate, filtered, and concentrated. Column chromatography (silica gel $30 \mathrm{~mL}, \mathrm{EtOAc} / \mathrm{EtOH}, 9 / 1$ to 8/2, v/v) of the residue afforded the product 20 as a mixture of stereoisomers and rotamers $(210 \mathrm{mg}, 288 \mu \mathrm{mol}, 63 \%)$ as a colorless oil. ${ }^{1} \mathrm{H}$ NMR $(500 \mathrm{MHz}$, $\left.\mathrm{CDCl}_{3}\right) \delta 7.52-6.92(\mathrm{~m}, 30 \mathrm{H}, \mathrm{H}$ aromatic $), 5.14,5.09,5.03,4.94(4 \mathrm{~d}, J=11.3 \mathrm{~Hz}, J=11.4 \mathrm{~Hz}, J=9.3 \mathrm{~Hz}$, $J=11.7 \mathrm{~Hz}, 1 \mathrm{H}, \mathrm{H}-1), 4.84-4.34\left(\mathrm{~m}, 6 \mathrm{H}, 3 \times \mathrm{OCH}_{2} \mathrm{Ph}\right), 4.29-3.55\left(\mathrm{~m}, 12 \mathrm{H}, \mathrm{H}-6,3 \times \mathrm{OCH}_{2} \mathrm{CH}_{3}, \mathrm{H}-3, \mathrm{H}-4\right.$, H-5, H-7, H-7'), 3.27-2.23 (m, 2H, $\left.\mathrm{PCH}_{2} \mathrm{P}\right), 2.00,1.97,1.94,1.92,1.91,1.90,1.87,\left(7 \mathrm{~s}, 3 \mathrm{H}, \mathrm{NHCOCH}_{3}\right)$, 1.36-1.15 (m, 9H, $\left.3 \times \mathrm{OCH}_{2} \mathrm{CH}_{3}\right) ;{ }^{31} \mathrm{P}$ NMR $\left(202 \mathrm{MHz}, \mathrm{CDCl}_{3}\right) \delta 31.3,30.8\left(2\right.$ broad s, $\left.\mathrm{CH}_{2} \mathrm{PCH}_{2} \mathrm{P}\right)$, 20.7, 20.6, 20.2, 20.1, 19.8, $19.6(6 \mathrm{~d}, J=4.7 \mathrm{~Hz}, J=5.7 \mathrm{~Hz}, J=2.1 \mathrm{~Hz}, J=2.9 \mathrm{~Hz}, J=5.8 \mathrm{~Hz}, J=3.6 \mathrm{~Hz}$, $\mathrm{CH}_{2} \mathrm{PCH}_{2} \mathrm{P}$ ); ESI-MS: Calcd for $\mathrm{C}_{37} \mathrm{H}_{49} \mathrm{NNaO}_{10} \mathrm{P}_{2}$ : 752.7327 [M + Na] $]^{+}$Found: 752.2; ESI-HRMS: Calcd for $\mathrm{C}_{37} \mathrm{H}_{49} \mathrm{NNaO}_{10} \mathrm{P}_{2}: 752.2724[\mathrm{M}+\mathrm{Na}]^{+}$Found: 752.2730 .

3.1.8. 2,6-Anhydro-1-deoxy-1-\{[(diethoxyphosphoryl)methyl](ethoxy)phosphoryl\}-D-glycero-Dgalacto-heptitol 21

To a solution of a mixture of the previous D-manno-hept-1-enitol 18 ( $476 \mathrm{mg}, 611 \mu \mathrm{mol})$ in ethanol $(28 \mathrm{~mL})$ under argon atmosphere was added a catalytic amount of palladium $10 \%$ on charcoal $(140 \mathrm{mg}$, $132 \mu \mathrm{mol})$. Then, the reaction mixture was purged with hydrogen gas and stirred overnight under 
hydrogen atmosphere. The resulting solution was degassed with argon atmosphere and it was filtered through a celite(C) (ThermoFisher, Kandel, Germany) patch which was rinsed with $10 \mathrm{~mL}$ of ethanol. The concentration under vacuum furnished the product 21 as a mixture of stereoisomers with a $\mathrm{R}$ configuration for the $\mathrm{C} 2$ stereogenic centre $\left(253 \mathrm{mg}, 602 \mu \mathrm{mol}\right.$, quant.) as a white solid. ${ }^{1} \mathrm{H} \mathrm{NMR}$

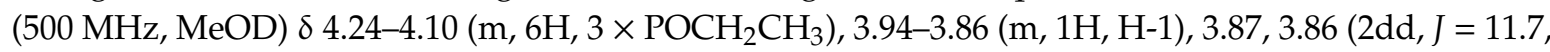
$2.3 \mathrm{~Hz}, J=11.3,2.4 \mathrm{~Hz}, 1 \mathrm{H}, \mathrm{H}-7), 3.72,3.71(2 \mathrm{~d}, J=3.0 \mathrm{~Hz}, 1 \mathrm{H}, \mathrm{H}-3), 3.66,3.65(2 \mathrm{dd}, J=11.3,6.7 \mathrm{~Hz}$, $\left.J=11.7,6.3 \mathrm{~Hz}, 1 \mathrm{H}, \mathrm{H}-7^{\prime}\right), 3.53(2 \mathrm{t}, J=9.4 \mathrm{~Hz}, 1 \mathrm{H}, \mathrm{H}-5), 3.49,3.48$ (2dd, $\left.J=9.4,3.0 \mathrm{~Hz}, 1 \mathrm{H}, \mathrm{H}-4\right), 3.26$, $3.23(2 \mathrm{ddd}, J=9.4,6.3,2.3 \mathrm{~Hz}, J=9.4,6.7,2.4 \mathrm{~Hz}, 1 \mathrm{H}, \mathrm{H}-6), 2.97-2.75\left(\mathrm{~m}, 2 \mathrm{H}, \mathrm{PCH}_{2} \mathrm{P}\right), 2.61-2.51(\mathrm{~m}$, 1H, H-1), 2.20-2.02 (m, 1H, H-1'), 1.35, $1.34\left(2 \mathrm{t}, J=7.0 \mathrm{~Hz}, 9 \mathrm{H}, 3 \times \mathrm{POCH}_{2} \mathrm{CH}_{3}\right)$; ${ }^{13} \mathrm{C} \mathrm{NMR}(126 \mathrm{MHz}$, MeOD) $\delta 82.33,82.28$ (C-6), $76.3(\mathrm{C}-4), 74.7,74.5(2 \mathrm{~d}, J=3.7 \mathrm{~Hz}, J=4.6 \mathrm{~Hz}, \mathrm{C}-2), 73.3,73.2(\mathrm{~d}, J=12.2 \mathrm{~Hz}$, $J=11.4 \mathrm{~Hz}, \mathrm{C}-3), 68.6,68.5(\mathrm{C}-5), 64.2,64.08,64.02,63.98,62.9,62.8\left(6 \mathrm{~d}, J=6.5 \mathrm{~Hz}, \mathrm{POCH}_{2} \mathrm{CH}_{3}\right), 63.06$, $63.00(\mathrm{C}-7), 33.5,33.4(2 \mathrm{~d}, J=99 \mathrm{~Hz}, \mathrm{C}-1), 28.6,28.4\left(2 \mathrm{dd}, J=135,83 \mathrm{~Hz}, \mathrm{PCH}_{2} \mathrm{P}\right), 16.83,16.79,16.7,16.6$ $\left(4 \mathrm{~d}, J=6.3 \mathrm{~Hz}, 3 \times \mathrm{POCH}_{2} \mathrm{CH}_{3}\right) ;{ }^{31} \mathrm{P} \mathrm{NMR}(202 \mathrm{MHz}, \mathrm{MeOD}) \delta 48.8,47.7(2 \mathrm{~d}, J=11.4 \mathrm{~Hz}, J=7.5 \mathrm{~Hz}$, $\left.\mathrm{CH}_{2} \mathrm{PCH}_{2} \mathrm{P}\right), 21.2,21.0\left(2 \mathrm{~d}, \mathrm{~J}=7.5 \mathrm{~Hz}, \mathrm{~J}=11.4 \mathrm{~Hz}, \mathrm{CH}_{2} \mathrm{PCH}_{2} \mathrm{P}\right)$; ESI-MS: Calcd for $\mathrm{C}_{28} \mathrm{H}_{59} \mathrm{NaO}_{20} \mathrm{P}_{4}$ : $862.7[2 \mathrm{M}-\mathrm{H}+\mathrm{Na}]^{+}$Found: 862.7; ESI-HRMS: Calcd for $\mathrm{C}_{14} \mathrm{H}_{30} \mathrm{NaO}_{10} \mathrm{P}_{2}: 443.1206[\mathrm{M}+\mathrm{Na}]^{+}$ Found: 443.1223.

3.1.9. 2,6-Anhydro-1-deoxy-1-\{[(diethoxyphosphoryl)methyl](ethoxy)phosphoryl\}-D-glycero-Dgulo-heptitol 22

To a solution of a mixture of the D-gulo-hept-1-enitol $19(720 \mathrm{mg}, 925 \mu \mathrm{mol})$ in ethanol (42 $\mathrm{mL})$ under argon atmosphere was added a catalytic amount of palladium $10 \%$ on charcoal $(210 \mathrm{mg}, 190 \mu \mathrm{mol})$. Then, the reaction mixture was purged with hydrogen gas and stirred overnight under hydrogen atmosphere. The resulting solution was degassed with argon atmosphere and it was filtered through a celite(C) patch. The concentration under vacuum furnished the product 22 as a mixture of stereoisomers with a R configuration for the C2 stereogenic centre $(377 \mathrm{mg}, 897 \mu \mathrm{mol}, 97 \%)$ as a white solid. ${ }^{1} \mathrm{H}$ NMR (500 MHz, MeOD) $\delta 4.24-4.08\left(\mathrm{~m}, 6 \mathrm{H}, 3 \times \mathrm{OCH}_{2} \mathrm{CH}_{3}\right), 3.87,3.86(2 \mathrm{dd}, J=11.9,2.2 \mathrm{~Hz}, \mathrm{~J}=11.8$, $1.4 \mathrm{~Hz}, 1 \mathrm{H}, \mathrm{H}-7), 3.64-3.50$ (m, 2H, H-7' , H-2), 3.38-3.22 (m, 3H, H-4, H-6, H-5), 3.11, 3.08 (2t, $J=9.0 \mathrm{~Hz}$, $J=9.1 \mathrm{~Hz}, 1 \mathrm{H}, \mathrm{H}-3), 2.99-2.74\left(\mathrm{~m}, 2 \mathrm{H}, \mathrm{PCH}_{2} \mathrm{P}\right), 2.57-2.40(\mathrm{~m}, 1 \mathrm{H}, \mathrm{H}-1), 2.26-2.16$ (m, 1H, H-1' $), 1.41-1.28$ $\left(\mathrm{m}, 9 \mathrm{H}, 3 \times \mathrm{OCH}_{2} \mathrm{CH}_{3}\right) ;{ }^{13} \mathrm{C}$ NMR $(126 \mathrm{MHz}, \mathrm{MeOD}) \delta 82.04,81.96(\mathrm{C}-6), 79.44,79.42(\mathrm{~d}, \mathrm{~J}=3,8 \mathrm{~Hz}$, $J=4.0 \mathrm{~Hz}, \mathrm{C}-4), 76.2,76.1(\mathrm{~d}, J=5.3 \mathrm{~Hz}, J=6.0 \mathrm{~Hz}, \mathrm{C}-2), 75.9,75.7(\mathrm{~d}, J=13.7 \mathrm{~Hz}, J=12.7 \mathrm{~Hz}, \mathrm{C}-3)$, 71.9, 71.8 (C-5), 64.1, 64.03, 63.99, 63.65, 62.81, $62.75(6 \mathrm{~d}, J=6.4 \mathrm{~Hz}, J=6.3 \mathrm{~Hz}, J=6.3 \mathrm{~Hz}, J=6.3 \mathrm{~Hz}$, $\left.J=6.5 \mathrm{~Hz}, J=6.6 \mathrm{~Hz}, \mathrm{OCH}_{2} \mathrm{CH}_{3}\right), 63.0,62.9(\mathrm{C}-7), 33.3,32.9(2 \mathrm{~d}, J=99.1 \mathrm{~Hz}, J=99.2 \mathrm{~Hz}, \mathrm{C}-1), 29.1,28.5$ $\left(2 \mathrm{dd}, J=135.0,24.4 \mathrm{~Hz}, J=135.0,24.9 \mathrm{~Hz}, \mathrm{PCH}_{2} \mathrm{P}\right), 16.9-16.53\left(\mathrm{~m}, 3 \times \mathrm{OCH}_{2} \mathrm{CH}_{3}\right) ;{ }^{31} \mathrm{P} \mathrm{NMR}(202 \mathrm{MHz}$, MeOD) $\delta 47.2,46.3\left(2 \mathrm{~d}, J=11.4 \mathrm{~Hz}, J=7.9 \mathrm{~Hz}, \mathrm{CH}_{2} \mathrm{PCH}_{2} \mathrm{P}\right), 19.8,19.6(\mathrm{~d}, J=7.9 \mathrm{~Hz}, J=11.4 \mathrm{~Hz}$, $\mathrm{CH}_{2} \mathrm{PCH}_{2} \mathrm{P}$ ); ESI-MS: Calcd for $\mathrm{C}_{28} \mathrm{H}_{59} \mathrm{NaO}_{20} \mathrm{P}_{4}: 862.7$ [2M - H + Na] ${ }^{+}$Found: 862.7; ESI-HRMS: Calcd for $\mathrm{C}_{14} \mathrm{H}_{31} \mathrm{O}_{10} \mathrm{P}_{2}: 421.1387[\mathrm{M}+\mathrm{H}]^{+}$Found: 421.1394 .

3.1.10. 3-Acetamido-2,6-anhydro-1,3-di-deoxy-1-\{[(diethoxyphosphoryl)methyl](ethoxy) phosphoryl\}-D-glycero-D-gulo-heptitol 23

To a solution of a mixture of the D-gluco-hept-1-enitol $20(210 \mathrm{mg}, 288 \mu \mathrm{mol})$ in ethanol $(14 \mathrm{~mL})$ under argon atmosphere was added a catalytic amount of palladium 10\% on charcoal (70 mg, $66 \mu \mathrm{mol})$. Then, the reaction mixture was purged with hydrogen gas and stirred overnight under hydrogen atmosphere. The resulting solution was degassed with argon atmosphere and it was filtered through a celite(C) patch. The concentration under vacuum furnished the product $\mathbf{2 3}$ as a mixture of stereoisomers with a R configuration for the $\mathrm{C} 2$ stereogenic centre $\left(132 \mathrm{mg}, 288 \mu \mathrm{mol}\right.$, quant.) as a white solid. ${ }^{1} \mathrm{H}$

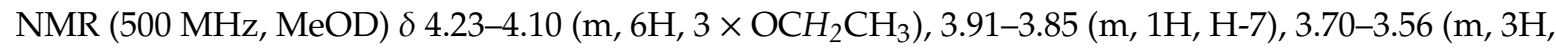
H-7' , H-3, H-2), 3.45-3.39 (m, 1H, H-4), 3.34-3.25 (m, 1H, H-5), 3.26 (ddd, J = 9.7, 5.8, 2.2 Hz, 1H, H-6), 3.05-2.71 (m, 2H, PCH $2 \mathrm{P}), 2.30-2.18(\mathrm{~m}, 1 \mathrm{H}, \mathrm{H}-1), 2.15-2.06\left(\mathrm{~m}, 1 \mathrm{H}, \mathrm{H}-1^{\prime}\right), 2.00\left(\mathrm{~s}, 3 \mathrm{H}, \mathrm{NHCOCH}_{3}\right)$, 1.37-1.32 (m, 9H, $\left.3 \times \mathrm{OCH}_{2} \mathrm{CH}_{3}\right) ;{ }^{13} \mathrm{C} \mathrm{NMR}(126 \mathrm{MHz}, \mathrm{MeOD}) \delta 173.93,173.89\left(\mathrm{NHCOCH}_{3}\right), 82.0$, $81.9(\mathrm{C}-6), 76.8(2 \mathrm{~d}, J=2.7 \mathrm{~Hz}, J=2.4 \mathrm{~Hz}, \mathrm{C}-4), 75.4,75.0(2 \mathrm{~d}, J=5.0 \mathrm{~Hz}, J=6.3 \mathrm{~Hz}, \mathrm{C}-2), 72.23,72.15$ 
(C-5), 64.1, 64.04, 63.98, 63.93, 62.85, $62.84(6 \mathrm{~d}, J=6.3 \mathrm{~Hz}, J=6.9 \mathrm{~Hz}, J=6.3 \mathrm{~Hz}, J=6.1 \mathrm{~Hz}, J=7.7 \mathrm{~Hz}$, $\left.J=7.7 \mathrm{~Hz}, 3 \times \mathrm{OCH}_{2} \mathrm{CH}_{3}\right), 62.92,62.88(\mathrm{C}-6), 57.7,57.6(\mathrm{~d}, J=13.6 \mathrm{~Hz}, J=14.2 \mathrm{~Hz}, \mathrm{C}-3), 33.8,33.4$ $(2 \mathrm{~d}, J=99.4 \mathrm{~Hz}, J=100.2 \mathrm{~Hz}, \mathrm{C}-1), 28.7,28.5\left(2 \mathrm{dd}, J=134.8,82.5 \mathrm{~Hz}, J=134.8,83.2 \mathrm{~Hz}, \mathrm{PCH}_{2} \mathrm{P}\right)$, 23.0, $22.9\left(\mathrm{NHCOCH}_{3}\right), 16.81,16.76,16.64,16.63(4 \mathrm{~d}, J=6.2 \mathrm{~Hz}, J=6.3 \mathrm{~Hz}, J=6.3 \mathrm{~Hz}, J=6.1 \mathrm{~Hz}$, $\left.3 \times \mathrm{OCH}_{2} \mathrm{CH}_{3}\right) ;{ }^{31} \mathrm{P}$ NMR $(202 \mathrm{MHz}, \mathrm{MeOD}) \delta 48.2,47.1\left(2 \mathrm{~d}, J=12.0 \mathrm{~Hz}, J=7.1 \mathrm{~Hz}, \mathrm{CH}_{2} P_{C H_{2} \mathrm{P}}\right.$, 21.3, $21.0\left(2 \mathrm{~d}, J=7.1 \mathrm{~Hz}, J=12.0 \mathrm{~Hz}, \mathrm{CH}_{2} \mathrm{PCH}_{2} P\right)$; ESI-MS: Calcd for $\mathrm{C}_{32} \mathrm{H}_{65} \mathrm{~N}_{2} \mathrm{NaO}_{20} \mathrm{P}_{4}$ : 944.8 [2M $\mathrm{H}+\mathrm{Na}]^{+}$Found: 944.7 , Calcd for $\mathrm{C}_{16} \mathrm{H}_{33} \mathrm{NNaO}_{10} \mathrm{P}_{2}: 484.4[\mathrm{M}+\mathrm{Na}]^{+}$Found: 484.1; ESI-HRMS: Calcd for $\mathrm{C}_{16} \mathrm{H}_{34} \mathrm{O}_{10} \mathrm{NP}_{2}: 462.1662[\mathrm{M}+\mathrm{H}]^{+}$Found: 462.1667 .

3.1.11. 3,4,5,7-Tetra-O-acetyl-2,6-anhydro-1-deoxy-1-\{[(diethoxyphosphoryl)methyl](ethoxy) phosphoryl\}-D-glycero-D-galacto-heptitol 24

To a solution of a mixture of the previous D-glycero-D-galacto-heptitol $21(235 \mathrm{mg}, 559 \mu \mathrm{mol})$ in pyridine $(9 \mathrm{~mL})$ cooled by an ice bath, was added dropwise acetic anhydride $(4.5 \mathrm{~mL}, 48 \mathrm{mmol})$. The reaction mixture was allowed to warm to room temperature. After stirring overnight at room temperature, the reaction is stopped by evaporation of the reactants. Column chromatography (silica gel $60 \mathrm{~mL}, \mathrm{CH}_{2} \mathrm{Cl}_{2} / \mathrm{EtOH}, 95 / 5$ to $9 / 1, v / v$ ) of the residue afforded 24 as a mixture of stereoisomers with a R configuration for the $\mathrm{C} 2$ stereogenic centre $(265 \mathrm{mg}, 450 \mu \mathrm{mol}, 80 \%)$ as a colorless oil. ${ }^{1} \mathrm{H}$ NMR $\left(500 \mathrm{MHz}, \mathrm{CDCl}_{3}\right) \delta 5.32,5.31(2$ broad d $, J=3.4 \mathrm{~Hz}, J=3.6 \mathrm{~Hz}, 1 \mathrm{H}, \mathrm{H}-3), 5.17,5.15(2 \mathrm{t}, J=10.0 \mathrm{~Hz}$, $1 \mathrm{H}, \mathrm{H}-5), 5.07,5.06(2 \mathrm{dd}, J=10.0,3.6 \mathrm{~Hz}, J=10.0,3.4 \mathrm{~Hz}, 1 \mathrm{H}, \mathrm{H}-4), 4.25-4.07$ (m, 9H, H-2, $3 \times$ $\left.\mathrm{POCH}_{2} \mathrm{CH}_{3}, \mathrm{H}-7, \mathrm{H}-7^{\prime}\right), 3.75,3.71$ (2ddd, $\left.J=10.0,7.0,2.3 \mathrm{~Hz}, J=10.0,6.3,2.4 \mathrm{~Hz}, 1 \mathrm{H}, \mathrm{H}-6\right), 2.70-2.35$ $\left(\mathrm{m}, 3 \mathrm{H}, 1.5 \times \mathrm{PCH}_{2} \mathrm{P}, \mathrm{H}-1\right), 2.17,2.08,2.04,1.96\left(4 \mathrm{~s}, 12 \mathrm{H}, 4 \times \mathrm{CH}_{3} \mathrm{CO}\right), 2.06-1.77\left(\mathrm{~m}, 1 \mathrm{H}, \mathrm{H}-1^{\prime}\right), 1.38-1.28$ $\left(\mathrm{m}, 9 \mathrm{H}, 3 \times \mathrm{POCH}_{2} \mathrm{CH}_{3}\right) ;{ }^{13} \mathrm{C}$ NMR $\left(126 \mathrm{MHz}, \mathrm{CDCl}_{3}\right) \delta 170.7,170.49,170.47,170.1,170.0,169.92$, $169.85\left(4 \times \mathrm{CH}_{3} \mathrm{CO}\right), 76.6,76.4(\mathrm{C}-6), 72.4,72.16(2 \mathrm{~d}, J=2.7 \mathrm{~Hz}, J=3.4 \mathrm{~Hz}, \mathrm{C}-2), 72.17,72.10(2 \mathrm{~d}$, $J=1.7 \mathrm{~Hz}, J=2.0 \mathrm{~Hz}, \mathrm{C}-4), 70.62,70.55(2 \mathrm{~d}, J=12.9 \mathrm{~Hz}, J=12.4 \mathrm{~Hz}, \mathrm{C}-3), 66.1,66.0(\mathrm{C}-5), 63.2,63.1$ $(\mathrm{C}-7), 62.8,62.62,62.60,62.36,61.58,61.56\left(6 \mathrm{~d}, J=6.5 \mathrm{~Hz}, 3 \times \mathrm{POCH}_{2} \mathrm{CH}_{3}\right), 32.2,31.1(2 \mathrm{~d}, J=98.5 \mathrm{~Hz}$, $J=99.7 \mathrm{~Hz}, \mathrm{C}-1), 29.3,28.9\left(2 \mathrm{dd}, J=134.5,83.1 \mathrm{~Hz}, J=134.6,81.9 \mathrm{~Hz}, \mathrm{PCH}_{2} \mathrm{P}\right), 20.86,20.84,20.83$, 20.69, $20.68\left(4 \times \mathrm{CH}_{3} \mathrm{CO}\right), 16.8-16.4\left(\mathrm{~m}, 3 \times \mathrm{POCH}_{2} \mathrm{CH}_{3}\right) ;{ }^{31} \mathrm{P} \mathrm{NMR}\left(202 \mathrm{MHz}, \mathrm{CDCl}_{3}\right) \delta 44.1,42.2(2 \mathrm{~d}$, $\left.J=9.8 \mathrm{~Hz}, J=5.9 \mathrm{~Hz}, \mathrm{CH}_{2} P \mathrm{PCH}_{2} \mathrm{P}\right), 19.9,19.7\left(\mathrm{~d}, J=9.8 \mathrm{~Hz}, J=5.9 \mathrm{~Hz}, \mathrm{CH}_{2} \mathrm{PCH}_{2} P\right)$; ESI-MS: Calcd for $\mathrm{C}_{44} \mathrm{H}_{75} \mathrm{NaO}_{28} \mathrm{P}_{4}: 1198.9$ [2M - H + Na] ${ }^{+}$Found: 1198.4; ESI-HRMS: Calcd for $\mathrm{C}_{22} \mathrm{H}_{39} \mathrm{O}_{14} \mathrm{P}_{2}: 589.1810$ $[\mathrm{M}+\mathrm{H}]^{+}$Found: 589.1818 .

3.1.12. 3,4,5,7-Tetra-O-acetyl-2,6-anhydro-1-deoxy-1-\{[(diethoxyphosphoryl)methyl](ethoxy) phosphoryl\}-D-glycero-D-gulo-heptitol 25

To a solution of a mixture of the previous D-glycero-D-gulo-heptitol $22(372 \mathrm{mg}, 885 \mu \mathrm{mol})$ in pyridine $(15 \mathrm{~mL})$ cooled by an ice bath, was added dropwise acetic anhydride $(7.5 \mathrm{~mL}, 79 \mathrm{mmol})$. The reaction mixture was allowed to warm to room temperature. After stirring overnight at room temperature, the reaction is stopped by evaporation of the reactants. Column chromatography (silica gel $80 \mathrm{~mL}, \mathrm{CH}_{2} \mathrm{Cl}_{2} / \mathrm{EtOH}, 95 / 5$ to $9 / 1, v / v$ ) of the residue afforded 25 as a mixture of stereoisomers with a R configuration for the $\mathrm{C} 2$ stereogenic centre $(503 \mathrm{mg}, 854 \mu \mathrm{mol}, 96 \%)$ as a colorless oil. ${ }^{1} \mathrm{H}$ NMR $\left(500 \mathrm{MHz}, \mathrm{CDCl}_{3}\right) \delta 5.14,5.13(2 \mathrm{t}, J=9.6 \mathrm{~Hz}, 1 \mathrm{H}, \mathrm{H}-4), 4.98,4.96(2 \mathrm{t}, J=9.6 \mathrm{~Hz}, 1 \mathrm{H}, \mathrm{H}-5), 4.87,4.86(2 \mathrm{t}$, $J=9.6 \mathrm{~Hz}, 1 \mathrm{H}, \mathrm{H}-3), 4.20-4.00\left(\mathrm{~m}, 8 \mathrm{H}, 3 \times \mathrm{OCH}_{2} \mathrm{CH}_{3}, \mathrm{H}-7, \mathrm{H}-7^{\prime}\right), 3.99-3.81(\mathrm{~m}, 1 \mathrm{H}, \mathrm{H}-2), 3.74,3.69$ (2ddd, $J=10.0,6.3,2.2 \mathrm{~Hz}, 1 \mathrm{H}, J=10.0,5.6,2.2 \mathrm{~Hz}, \mathrm{H}-6), 2.70-2.39\left(\mathrm{~m}, 2 \mathrm{H}, \mathrm{PCH}_{2} \mathrm{P}\right), 2.32-1.86(\mathrm{~m}, 2 \mathrm{H}$, $\left.\mathrm{H}-1, \mathrm{H}_{-1}{ }^{\prime}\right), 2.03,2.01,2.00,1.98,1.95,1.94\left(6 \mathrm{~s}, 12 \mathrm{H}, 4 \times \mathrm{CH}_{3} \mathrm{CO}\right), 1.34-1.25\left(\mathrm{~m}, 9 \mathrm{H}, 3 \times \mathrm{OCH}_{2} \mathrm{CH}_{3}\right) ;{ }^{13} \mathrm{C}$ NMR $\left(126 \mathrm{MHz}, \mathrm{CDCl}_{3}\right) \delta 170.5,170.3,170.2,169.7,169.6,169.5\left(4 \times \mathrm{CH}_{3} \mathrm{CO}\right), 76.0,75.8(\mathrm{C}-6), 74.0,73.9$ $(2 \mathrm{~d}, J=2.2 \mathrm{~Hz}, J=1.7 \mathrm{~Hz}, \mathrm{C}-4), 73.4,73.2(2 \mathrm{~d}, J=4.0 \mathrm{~Hz}, J=6.5 \mathrm{~Hz}, \mathrm{C}-2), 72.2,72.1(\mathrm{~d}, J=14.6 \mathrm{~Hz}$, $J=13.8 \mathrm{~Hz}, \mathrm{C}-3), 68.6,68.5(\mathrm{C}-5), 62.7,62.5,62.4,62.3,61.4(5 \mathrm{~d}, J=6.3 \mathrm{~Hz}, J=6.1 \mathrm{~Hz}, J=4.5 \mathrm{~Hz}$, $\left.J=6.4 \mathrm{~Hz}, \mathrm{~Hz}, J=6.5 \mathrm{~Hz}, 3 \times \mathrm{OCH}_{2} \mathrm{CH}_{3}\right), 62.5,62.4(\mathrm{C}-7), 32.4,31.5(2 \mathrm{~d}, J=98.0 \mathrm{~Hz}, J=100.0 \mathrm{~Hz}, \mathrm{C}-1)$, 29.5, $28.9\left(2 \mathrm{dd}, J=134.5,83.8 \mathrm{~Hz}, J=134.9,81.7 \mathrm{~Hz}, \mathrm{PCH}_{2} \mathrm{P}\right), 20.7,20.6\left(4 \times \mathrm{CH}_{3} \mathrm{CO}\right), 16.6-16.3(\mathrm{~m}, 3 \times$ $\left.\mathrm{OCH}_{2} \mathrm{CH}_{3}\right) ;{ }^{31} \mathrm{P}$ NMR $\left(202 \mathrm{MHz}, \mathrm{CDCl}_{3}\right) \delta 43.9,42.1\left(2 \mathrm{~d}, J=9.0 \mathrm{~Hz}, J=3.1 \mathrm{~Hz}, \mathrm{CH}_{2} P_{C H} \mathrm{P}\right), 19.9,19.7$ 
$\left(2 \mathrm{~d}, J=9.0 \mathrm{~Hz}, J=3.1 \mathrm{~Hz}, \mathrm{CH}_{2} \mathrm{PCH}_{2} P\right)$; ESI-MS: Calcd for $\mathrm{C}_{44} \mathrm{H}_{75} \mathrm{NaO}_{28} \mathrm{P}_{4}: 1198.9\left[2 \mathrm{M}-\mathrm{H}+\mathrm{Na}^{+}\right.$ Found: 1198.4; ESI-HRMS: Calcd for $\mathrm{C}_{22} \mathrm{H}_{39} \mathrm{O}_{14} \mathrm{P}_{2}$ : 589.1810 [M + H] ${ }^{+}$Found: 589.1820.

3.1.13. 3-Acetamido-4,5,7-tri-O-acetyl-2,6-anhydro-1,3-di-deoxy-1-\{[(diethoxyphosphoryl) methyl](ethoxy)phosphoryl\}-D-glycero-D-gulo-heptitol 26

To a solution of a mixture of the previous D-glycero-D-gulo-heptitol $23(132 \mathrm{mg}, 288 \mu \mathrm{mol})$ in pyridine $(5 \mathrm{~mL})$ cooled by an ice bath, was added dropwise acetic anhydride $(2.5 \mathrm{~mL}, 26.4 \mathrm{mmol})$. The reaction mixture was allowed to warm to room temperature. After stirring overnight at room temperature, the reaction is stopped by evaporation of the reactants. Column chromatography (silica gel $30 \mathrm{~mL}, \mathrm{CH}_{2} \mathrm{Cl}_{2} / \mathrm{EtOH}, 95 / 5$ to $9 / 1, v / v$ ) of the residue afforded 26 as a mixture of stereoisomers with a R configuration for the $\mathrm{C} 2$ stereogenic centre $(124 \mathrm{mg}, 211 \mu \mathrm{mol}, 73 \%)$ as a colorless oil. ${ }^{1} \mathrm{H}$ NMR $\left(500 \mathrm{MHz}, \mathrm{CDCl}_{3}\right) \delta 6.27,6.20\left(2 \mathrm{~d}, J=9.3 \mathrm{~Hz}, J=9.5 \mathrm{~Hz}, 1 \mathrm{H}, \mathrm{NHCOCH}_{3}\right), 5.09-4.98(\mathrm{~m}, 2 \mathrm{H}, \mathrm{H}-5, \mathrm{H}-4)$, 4.25-3.99 (m, 9H, $\left.3 \times \mathrm{OCH}_{2} \mathrm{CH}_{3}, \mathrm{H}-7, \mathrm{H}-7^{\prime}, \mathrm{H}-3\right), 3.92,3.86$ (2ddd, $J=11.9,9.5,2.2 \mathrm{~Hz}, J=12.1,9.3$, $2.2 \mathrm{~Hz}, 1 \mathrm{H}, \mathrm{H}-2), 3.75-3.67$ (m, 1H, H-6), 2.66-2.01 (m, 4H, H-1, H-1' $\left.{ }^{\prime} \mathrm{PCH}_{2} \mathrm{P}\right), 2.07,2.06,2.02,2.01$, $2.00\left(5 \mathrm{~s}, 9 \mathrm{H}, 3 \times \mathrm{OCOCH}_{3}\right), 1.94\left(\mathrm{~s}, 3 \mathrm{H}, \mathrm{NHCOCH}_{3}\right), 1.38-1.30\left(\mathrm{~m}, 9 \mathrm{H}, 3 \times \mathrm{OCH}_{2} \mathrm{CH}_{3}\right) ;{ }^{13} \mathrm{C} \mathrm{NMR}$ $\left(126 \mathrm{MHz}, \mathrm{CDCl}_{3}\right) \delta 171.4,171.3,170.75,170.74,170.67,170.6,169.5\left(3 \times \mathrm{OCOCH}_{3}, \mathrm{NHCOCH}_{3}\right), 76.1$, $75.9(\mathrm{C}-6), 74.7,74.0(2 \mathrm{~d}, J=3.6 \mathrm{~Hz}, J=5.8 \mathrm{~Hz}, \mathrm{C}-2), 74.33,74.29(2 \mathrm{~d}, J=2.4 \mathrm{~Hz}, J=3.4 \mathrm{~Hz}, \mathrm{C}-4), 68.7$, $68.6(\mathrm{C}-5), 63.0,62.64,62.56,62.54,61.5,61.4\left(6 \mathrm{~d}, J=6.5 \mathrm{~Hz}, 3 \times \mathrm{OCH}_{2} \mathrm{CH}_{3}\right), 62.8,62.52(\mathrm{C}-7), 54.31$, $54.25(2 \mathrm{~d}, J=13.5 \mathrm{~Hz}, J=14.8 \mathrm{~Hz}, \mathrm{C}-3), 32.8,32.0(\mathrm{~d}, J=98.1 \mathrm{~Hz}, J=99.4 \mathrm{~Hz}, \mathrm{C}-1), 29.3,28.7(2 \mathrm{dd}$, $\left.J=134.5,83.6 \mathrm{~Hz}, J=135.1,81.6 \mathrm{~Hz}, \mathrm{CH}_{2} \mathrm{PCH}_{2} \mathrm{P}\right), 23.3\left(\mathrm{NHCOCH}_{3}\right), 20.84,20.81,20.79,20.74,20.73(3 \times$ $\left.\mathrm{OCOCH}_{3}\right), 16.7-16.4\left(3 \times \mathrm{OCH}_{2} \mathrm{CH}_{3}\right) ;{ }^{31} \mathrm{P} \mathrm{NMR}\left(202 \mathrm{MHz}, \mathrm{CDCl}_{3}\right) \delta 44.8,43.0(2 \mathrm{~d}, J=8.0 \mathrm{~Hz}, J=1.4 \mathrm{~Hz}$, $\left.\mathrm{CH}_{2} \mathrm{PCH}_{2} \mathrm{P}\right), 19.8,19.6\left(2 \mathrm{~d}, J=8.0 \mathrm{~Hz}, J=1.4 \mathrm{~Hz}, \mathrm{CH}_{2} \mathrm{PCH}_{2} P\right)$; ESI-MS: Calcd for $\mathrm{C}_{22} \mathrm{H}_{39} \mathrm{NNaO}_{13} \mathrm{P}_{2}$ : $610.5[\mathrm{M}+\mathrm{Na}]^{+}$Found: 610.1, Calcd for $\mathrm{C}_{44} \mathrm{H}_{77} \mathrm{~N}_{2} \mathrm{NaO}_{26} \mathrm{P}_{4}: 1197.0[2 \mathrm{M}-\mathrm{H}+\mathrm{Na}]^{+}$Found: 1196.5; ESI-HRMS: Calcd for $\mathrm{C}_{22} \mathrm{H}_{40} \mathrm{NO}_{13} \mathrm{P}_{2}: 588.1969[\mathrm{M}+\mathrm{H}]^{+}$Found: 588.1991.

3.1.14. Sodium \{[oxido(2,6-anhydro-1-deoxy-D-glycero-D-galacto-heptityl)phosphoryl]methyl\} phosphonate 27

To a solution of a mixture of the previous D-glycero-D-galacto-heptitol 24 (53.5 mg, $90 \mu \mathrm{mol})$ in anhydrous dichloromethane $(2 \mathrm{~mL})$ cooled by an ice bath, was added dropwise bromotrimethylsilane $(142 \mu \mathrm{L}, 1.08 \mathrm{mmol})$. The reaction mixture was allowed to warm to room temperature. After stirring overnight at room temperature, the reaction is stopped by evaporation of the reactants. Then the residue was dissolved in water $(4 \mathrm{~mL})$. Sodium hydoxide $(28.8 \mathrm{mg}, 720 \mu \mathrm{mol})$ was added to the reaction mixture. After $16 \mathrm{~h}$ of stirring at room temperature, the reaction was stopped with addition of cation exchange resin (DOWEX@ 50WX8 resin, $\mathrm{H}^{+}$form, ACROS, Geel, Belgium). The resin was removed by filtration through a millipore filter $(45 \mu \mathrm{m}$, AIT, Houilles, France). The aqueous solution was freeze dried. The resulting solid $(48 \mathrm{mg})$ was solubilized in water $(0.48 \mathrm{~mL})$. To this solution was added absolute ethanol $(4.8 \mathrm{~mL})$. After $16 \mathrm{~h}$ at room temperature, the resulting solid was recovered and rinsed with absolute ethanol. It was solubilized in water and freeze dried to afford the phosphonate $\mathbf{2 7}$ as a white solid (34.6 mg, $86 \mu \mathrm{mol}, 95 \%) .{ }^{1} \mathrm{H}$ NMR $\left(500 \mathrm{MHz}, \mathrm{CDCl}_{3}\right) \delta 4.03(\mathrm{td}, J=9.3,3.9 \mathrm{~Hz}, 1 \mathrm{H}, \mathrm{H}-2)$, $3.97(\mathrm{dd}, J=12.3,2.0 \mathrm{~Hz}, 1 \mathrm{H}, \mathrm{H}-7), 3.94(\mathrm{~d}, J=3.2 \mathrm{~Hz}, 1 \mathrm{H}, \mathrm{H}-3), 3.78\left(\mathrm{dd}, J=12.3,6.3 \mathrm{~Hz}, 1 \mathrm{H}, \mathrm{H}-7^{\prime}\right)$, $3.76(\mathrm{dd}, J=9.6,3.2 \mathrm{~Hz}, 1 \mathrm{H}, \mathrm{H}-4), 3.63(\mathrm{t}, J=9.6 \mathrm{~Hz}, 1 \mathrm{H}, \mathrm{H}-5), 3.45(\mathrm{ddd}, J=9.6,6.3,2.0 \mathrm{~Hz}, 1 \mathrm{H}, \mathrm{H}-6)$, 2.57-2.34 (m, 3H, PCH $\left.{ }_{2} \mathrm{P}, \mathrm{H}-1\right), 2.14\left(\mathrm{td}, J=16.3,3.9 \mathrm{~Hz}, 1 \mathrm{H}, \mathrm{H}-1^{\prime}\right) ;{ }^{13} \mathrm{C}$ NMR $\left(126 \mathrm{MHz}, \mathrm{CDCl}_{3}\right) \delta$ 80.0 (C-6), $74.2(\mathrm{C}-4), 73.6$ (d, $J=2.8 \mathrm{~Hz}, \mathrm{C}-2), 71.8(\mathrm{~d}, J=10.5 \mathrm{~Hz}, \mathrm{C}-3), 67.0$ (C-5), 61.3 (C-7), 32.2 $(\mathrm{d}, J=96.5 \mathrm{~Hz}, \mathrm{C}-1), 30.9\left(\mathrm{dd}, J=122.1,80.7 \mathrm{~Hz}, \mathrm{PCH}_{2} \mathrm{PO}\right) ;{ }^{31} \mathrm{P} \mathrm{NMR}\left(202 \mathrm{MHz}, \mathrm{CDCl}_{3}\right) \delta 36.05(\mathrm{~d}$, $\left.J=4.5 \mathrm{~Hz}, \mathrm{CH}_{2} \mathrm{PCH}_{2} \mathrm{P}\right), 11.75-11.65\left(\mathrm{~m}, \mathrm{CH}_{2} \mathrm{PCH}_{2} P\right) ;{ }^{31} \mathrm{P}$ NMR non decoupled $\left(202 \mathrm{MHz}, \mathrm{CDCl}_{3}\right) \delta$ 36.50-35.73 (m, $\left.\mathrm{CH}_{2} \mathrm{PCH}_{2} \mathrm{P}\right), 11.70\left(\mathrm{td}, J=18.5,4.5 \mathrm{~Hz}, \mathrm{CH}_{2} \mathrm{PCH}_{2} P\right.$ ); ESI-MS (mobile phase gradient ACN $/ \mathrm{H}_{2} \mathrm{O}+0.1 \%$ formic acid): Calcd for $\mathrm{C}_{8} \mathrm{H}_{19} \mathrm{O}_{10} \mathrm{P}_{2}: 337.0[\mathrm{M}+\mathrm{H}]^{+}$Found: 337.0; ESI-HRMS: Calcd for $\mathrm{C}_{8} \mathrm{H}_{19} \mathrm{O}_{10} \mathrm{P}_{2}$ : $337.0453[\mathrm{M}+\mathrm{H}]^{+}$Found: 337.0443 . 
3.1.15. Sodium \{[oxido(2,6-anhydro-1-deoxy-D-glycero-D-gulo-heptityl)phosphoryl]methyl\} phosphonate 28

To a solution of a mixture of the previous D-glycero-D-gulo-heptitol $25(63.5 \mathrm{mg}, 116 \mu \mathrm{mol})$ in anhydrous dichloromethane $(2 \mathrm{~mL})$ cooled by an ice bath, was added dropwise bromotrimethylsilane $(183 \mu \mathrm{L}, 1.39 \mathrm{mmol})$. The reaction mixture was allowed to warm to room temperature. After stirring overnight at room temperature, the reaction is stopped by evaporation of the reactants. Then the residue was dissolved in water $(4 \mathrm{~mL})$. Sodium hydoxide $(37 \mathrm{mg}, 925 \mu \mathrm{mol})$ was added to the reaction mixture. After $16 \mathrm{~h}$ of stirring at room temperature, the reaction was stopped with addition of cation exchange resin (DOWEXC $50 \mathrm{WX} 8$ resin, $\mathrm{H}^{+}$form). The resin was removed by filtration through a millipore filter $(45 \mu \mathrm{m})$. The aqueous solution was freeze dried. The resulting solid $(62 \mathrm{mg})$ was solubilized in water $(0.62 \mathrm{~mL})$. To this solution was added absolute ethanol $(6.2 \mathrm{~mL})$. After $16 \mathrm{~h}$ at room temperature, the resulting solid was recovered and rinsed with absolute ethanol. It was solubilized in water and freeze dried to afford the phosphonate 28 as a white solid ( $43 \mathrm{mg}, 107 \mu \mathrm{mol}, 92 \%) .{ }^{1} \mathrm{H}$ NMR (500 MHz, $\left.\mathrm{D}_{2} \mathrm{O}\right) \delta 3.93(\mathrm{~d}, J=12.3 \mathrm{~Hz}, 1 \mathrm{H}, \mathrm{H}-7), 3.74\left(\mathrm{dd}, J=12.3,4.3 \mathrm{~Hz}, 1 \mathrm{H}, \mathrm{H}-7^{\prime}\right), 3.68$ (qd, $J=9.4,2.5 \mathrm{~Hz}, 1 \mathrm{H}, \mathrm{H}-2), 3.52(\mathrm{t}, J=9.4 \mathrm{~Hz}, 1 \mathrm{H}, \mathrm{H}-4), 3.48-3.40(\mathrm{~m}, 2 \mathrm{H}, \mathrm{H}-5, \mathrm{H}-6), 3.26(\mathrm{t}, J=9.4 \mathrm{~Hz}, 1 \mathrm{H}$, $\mathrm{H}-3), 2.33-2.01\left(\mathrm{~m}, 4 \mathrm{H}, \mathrm{H}-1, \mathrm{H}-\mathrm{1}^{\prime}, \mathrm{PCH}_{2} \mathrm{P}\right) ;{ }^{13} \mathrm{C}$ NMR $\left(126 \mathrm{MHz}, \mathrm{D}_{2} \mathrm{O}\right) \delta 79.5$ (C-6), $77.2(\mathrm{C}-4), 75.6(\mathrm{~d}$, $J=4.3 \mathrm{~Hz}, \mathrm{C}-2), 74.5$ (d, $J=11.2 \mathrm{~Hz}, \mathrm{C}-3), 69.8(\mathrm{C}-5), 60.9$ (C-7), 33.71 (d, $J=96.0 \mathrm{~Hz}, \mathrm{C}-1), 32.38$ (dd, $\left.J=119.5,79.8 \mathrm{~Hz}, \mathrm{PCH}_{2} \mathrm{PO}\right) ;{ }^{31} \mathrm{P}$ NMR $\left(202 \mathrm{MHz}, \mathrm{D}_{2} \mathrm{O}\right) \delta 34.72\left(\mathrm{~d}, J=6.1 \mathrm{~Hz}, \mathrm{CH}_{2} \mathrm{PCH}_{2} \mathrm{P}\right), 15.25(\mathrm{~d}$, $\left.J=6.1 \mathrm{~Hz}, \mathrm{CH}_{2} \mathrm{PCH}_{2} P\right) ;{ }^{31} \mathrm{P}$ NMR non decoupled $\left(202 \mathrm{MHz}, \mathrm{D}_{2} \mathrm{O}\right) \delta 35.97-32.61\left(\mathrm{~m}, \mathrm{CH}_{2} \mathrm{PCH}_{2} \mathrm{P}\right), 15.22$ (td, $J=19.3,6.1 \mathrm{~Hz}, \mathrm{CH}_{2} \mathrm{PCH}_{2} P$ ); ESI-MS (mobile phase gradient $\mathrm{ACN} / \mathrm{H}_{2} \mathrm{O}+0.1 \%$ formic acid): Calcd for $\mathrm{C}_{8} \mathrm{H}_{19} \mathrm{O}_{10} \mathrm{P}_{2}: 337.0[\mathrm{M}+\mathrm{H}]^{+}$Found: 337.0; ESI-HRMS: Calcd for $\mathrm{C}_{8} \mathrm{H}_{19} \mathrm{O}_{10} \mathrm{P}_{2}: 337.0453[\mathrm{M}+\mathrm{H}]^{+}$ Found: 337.0448 .

3.1.16. Sodium \{[oxido(3-acetamido-2,6-anhydro-1,3-di-deoxy-D-glycero-D-gulo-heptityl) phosphoryl]methyl phosphonate 29

To a solution of a mixture of the previous D-glycero-D-gulo-heptitol $26(51 \mathrm{mg}, 87 \mu \mathrm{mol})$ in anhydrous dichloromethane $(2 \mathrm{~mL})$ cooled by an ice bath, was added dropwise bromotrimethylsilane $(137 \mu \mathrm{L}$, $1.04 \mathrm{mmol}$ ). The reaction mixture was allowed to warm to room temperature. After stirring overnight at room temperature, the reaction is stopped by evaporation of the reactants. Then the residue was dissolved in water $(4 \mathrm{~mL})$. Sodium hydoxide $(27.8 \mathrm{mg}, 695 \mu \mathrm{mol})$ was added to the reaction mixture. After $16 \mathrm{~h}$ of stirring at room temperature, the reaction was stopped with addition of cation exchange resin (DOWEX@ 50WX8 resin, $\mathrm{H}^{+}$form). The resin was removed by filtration through a millipore filter $(45 \mu \mathrm{m})$. The aqueous solution was freeze dried. The resulting solid $(51 \mathrm{mg})$ was solubilized in water $(0.51 \mathrm{~mL})$. To this solution was added absolute ethanol $(5.1 \mathrm{~mL})$. After $16 \mathrm{~h}$ at room temperature, the resulting solid was recovered and rinsed with absolute ethanol. It was solubilized in water and freeze dried to afford the phosphonate 29 as an orange solid (32.9 $\mathrm{mg}, 74 \mu \mathrm{mol}, 85 \%)$. ${ }^{1} \mathrm{H}$ NMR $\left(500 \mathrm{MHz}, \mathrm{D}_{2} \mathrm{O}\right) \delta 3.95(\mathrm{~d}, J=12.4 \mathrm{~Hz}, 1 \mathrm{H}, \mathrm{H}-7), 3.78\left(\mathrm{dd}, J=12.4,5.1 \mathrm{~Hz}, 1 \mathrm{H}, \mathrm{H}-7^{\prime}\right), 3.77-3.71(\mathrm{~m}, 1 \mathrm{H}$, $\mathrm{H}-2), 3.67(\mathrm{t}, J=9.3 \mathrm{~Hz}, 1 \mathrm{H}, \mathrm{H}-3), 3.56(\mathrm{t}, J=9.3 \mathrm{~Hz}, 1 \mathrm{H}, \mathrm{H}-4), 3.50(\mathrm{t}, J=9.3 \mathrm{~Hz}, 1 \mathrm{H}, \mathrm{H}-5), 3.45(\mathrm{dd}$, $J=9.3,5.1 \mathrm{~Hz}, 1 \mathrm{H}, \mathrm{H}-6), 2.27\left(\mathrm{dt}, J=19.2,15.7 \mathrm{~Hz}, 1 \mathrm{H}, 0.5 \times \mathrm{PCH}_{2} \mathrm{P}\right), 2.19-2.05\left(\mathrm{~m}, 1 \mathrm{H}, 0.5 \times \mathrm{PCH}_{2} \mathrm{P}\right)$, $2.10\left(\mathrm{~s}, 3 \mathrm{H}, \mathrm{NHCOCH}_{3}\right), 2.05-1.89\left(\mathrm{~m}, 2 \mathrm{H}, \mathrm{H}-1, \mathrm{H}-\mathrm{1}^{\prime}\right) ;{ }^{13} \mathrm{C} \mathrm{NMR}\left(126 \mathrm{MHz}, \mathrm{D}_{2} \mathrm{O}\right) \delta 174.7\left(\mathrm{NHCOCH}_{3}\right)$, 79.4 (C-6), 75.4 (C-4), 74.5 (d, J = 5.1 Hz, C-2), 69.9 (C-5), 60.8 (C-7), 56.5 (d, J = 13.2 Hz, C-3), 33.4 $(\mathrm{d}, J=96.4 \mathrm{~Hz}, \mathrm{C}-1), 32.0\left(\mathrm{dd}, J=119.8,79.3 \mathrm{~Hz}, \mathrm{PCH}_{2} \mathrm{PO}\right), 22.3\left(\mathrm{NHCOCH}_{3}\right) ;{ }^{31} \mathrm{P} \mathrm{NMR}(202 \mathrm{MHz}$, $\left.\mathrm{D}_{2} \mathrm{O}\right) \delta 34.08\left(\mathrm{~d}, J=7.5 \mathrm{~Hz}, \mathrm{CH}_{2} P \mathrm{PCH}_{2} \mathrm{P}\right), 15.76\left(\mathrm{~d}, J=7.5 \mathrm{~Hz}, \mathrm{CH}_{2} \mathrm{PCH}_{2} P\right) ;{ }^{31} \mathrm{P}$ NMR non decoupled $\left(202 \mathrm{MHz}, \mathrm{D}_{2} \mathrm{O}\right) \delta 34.3-33.8\left(\mathrm{~m}, \mathrm{CH}_{2} P \mathrm{PCH}_{2} \mathrm{P}\right), 15.74\left(\mathrm{td}, J=19.4,7.5 \mathrm{~Hz}, \mathrm{CH}_{2} \mathrm{PCH}_{2} P\right.$ ); ESI-MS (mobile phase gradient $\mathrm{ACN} / \mathrm{H}_{2} \mathrm{O}+0.1 \%$ formic acid): Calcd for $\mathrm{C}_{10} \mathrm{H}_{22} \mathrm{NO}_{10} \mathrm{P}_{2}: 378.1[\mathrm{M}+\mathrm{H}]^{+}$Found: 378.1; ESI-HRMS: Calcd for $\mathrm{C}_{10} \mathrm{H}_{22} \mathrm{NO}_{10} \mathrm{P}_{2}: 378.0719[\mathrm{M}+\mathrm{H}]^{+}$Found: 378.0709 . 


\subsection{Enzyme Assays}

\subsubsection{DLODP Assay}

Microsomal membranes containing DLODP were prepared from rat liver (APAFIS\#20275$2019041612268204 \mathrm{v} 4)$ as previously described [46]. Membranes (8.5 mg) were solubilized in $20 \mathrm{mM}$ MES pH 6.2, $200 \mathrm{mM} \mathrm{NaCl}, 1 \mathrm{mM} \mathrm{CoCl} 2,10 \%$ glycerol, $12.5 \mathrm{mg}$ of NP-40 for $1 \mathrm{~h}$ at $4{ }^{\circ} \mathrm{C}$. The solution was centrifuged at $100,000 \mathrm{gAV}$ for $45 \mathrm{~min}$ at $4{ }^{\circ} \mathrm{C}$. The supernatant containing solubilized DLODP was collected and stored at $-150{ }^{\circ} \mathrm{C}$. DLODP assay was performed in a final mixture of $20 \mathrm{mM}$ MES pH 5.5, $150 \mathrm{mM} \mathrm{NaCl}, 1 \mathrm{mM} \mathrm{CoCl} 2,10 \%$ glycerol, $0.2 \%$ NP-40, $20 \mu \mathrm{g}$ of solubilized membrane, metabolically radiolabeled dolichol-linked oligosaccharide $\left(3.0 \times 10^{4} \mathrm{cpm}\right), 0.1 \mathrm{mM}$ kifunensin and

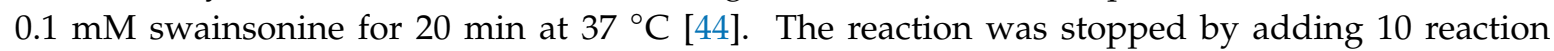
volumes of cold distilled water. The solution was added to coupled Dowex $50 \mathrm{WX} 4\left(\mathrm{H}^{+}\right.$form, Sigma Aldrich, SARL, Saint-Quentin Fallavier, France) and Dowex $1 \times 8$ (formate form, Sigma Aldrich SARL, Saint-Quentin Fallavier, France) ion-exchangers. The exchangers were washed with 12 column volumes of distilled water, then the Dowex $1 \times 8$ was eluted with 5 column volumes of $3 \mathrm{M}$ formic acid. The eluate was dried and radioactivity determined by scintillation counting.

\subsubsection{Alkaline Phosphatase Assay}

Alkaline phosphatase (524572, Sigma Aldrich SARL, Saint-Quentin Fallavier, France) activity was determined by measuring the quantity of $\left[{ }^{14} \mathrm{C}\right]$ glucose released from $\left[{ }^{14} \mathrm{C}\right]$ glucose-1-phosphate (295 mCi/mmol, PerkinElmer Life Sciences, Boston, MA, USA). The assay was performed in a final mixture of $50 \mathrm{mM}$ Tris- $\mathrm{HCl} \mathrm{pH} 8.5,1 \mathrm{mM} \mathrm{MgCl}_{2}, 0.1 \mathrm{mM} \mathrm{ZnC}_{4} \mathrm{H}_{6} \mathrm{O}_{4}, 5 \mu \mathrm{M}\left[{ }^{14} \mathrm{C}\right]$ glucose-1-phosphate $\left(3.5 \times 10^{4} \mathrm{cpm}\right)$ and $0-10 \mathrm{mM}$ of water soluble compounds. After incubation at $37^{\circ} \mathrm{C}$ for $30 \mathrm{~min}$, the mixture was kept on ice and $10 \mathrm{mM}$ sodium orthovanadate was added to stop the reaction. The solution was then applied to combined Dowex 50WX4 ( $\mathrm{H}^{+}$form) and Dowex $1 \times 8$ (formate) columns, and the elution and water wash fractions containing $\left[{ }^{14} \mathrm{C}\right]$ glucose were dried and quantified by scintillation counting.

\section{Conclusions}

New non hydrolysable glycosyl diphosphate mimics displaying an original $\beta-1 C$-(phosphino)phosphonate structure were synthetized according to a general convergent strategy. The key steps of the synthesis involved the addition of conveniently protected $\delta$-hexonolactones derived from Man, Glc and GlcNAc onto the dianion of diethyl ethoxy(methyl)phosphinylmethyl phosphonate, followed by dehydration and subsequent reduction of the resulting double bond leading to pure $\beta$ stereochemistry. The synthetized compounds were tested for their ability to inhibit DLO diphosphatase and phosphatase activities. Whereas the compounds had no effect on the $\mathrm{Co}^{2+}$-activated DLO diphosphatase at a concentration of $1 \mathrm{mM}$, complex concentration-dependent activatory and inhibitory effects on alkaline phosphatase were noted. These compounds are undergoing further exploration as potential modifiers of the enzymatic metabolism of phospho-compounds.

Supplementary Materials: The following are available online. Experimental procedures for compounds 11-13; NMR spectra for compounds 11-29.

Author Contributions: Conceptualization, M.B., S.-J.P., P.B., S.E.H.M. and C.G.-P.; Data curation, M.B., S.-J.P., S.E.H.M. and C.G.-P.; Funding acquisition, P.B., S.E.H.M. and C.G.-P.; Investigation, M.B. and S.-J.P.; Methodology, M.B., S.-J.P., P.B., S.E.H.M. and C.G.-P.; Project administration, P.B., S.E.H.M. and C.G.-P.; Supervision, P.B., S.E.H.M. and C.G.-P.; Writing-original draft, M.B., S.-J.P., S.E.H.M. and C.G.-P.; Writing-review \& editing, M.B., S.-J.P., P.B., S.E.H.M. and C.G.-P. All authors have read and agreed to the published version of the manuscript.

Funding: This research was funded by la Fondation pour la Recherche Médicale (FRM: DCM20121225751 and by the European Union FP6-Coordination Action EUROGLYCANET (LSHM-CT-2005-512131), the E-Rare-2 Joint Transnational Call 2011 (EURO-CDG), the Agence Nationale de la Recherche (ANR) (ANR-18-CE44-0007) and institutional funding from INSERM, CNRS and the Ministère de l'Enseignement Supérieur et de la Recherche. 
Acknowledgments: Assia Hessani (Université de Paris) is gratefully acknowledged for assistance with low resolution and high resolution mass spectra analyses. This work has benefited from the facilities and expertise of "Service HPLC-MASSE », Institut de Chimie des Substances Naturelles, Centre de Recherche de Gif sur Yvette, CNRS, 91198, Gif sur Yvette, France. The NMR experiments were performed thanks to NMR facilities of UMR 8601.

Conflicts of Interest: The authors declare no conflict of interest.

\section{References}

1. Tytgat, H.L.P.; Lebeer, S. The sweet tooth of bacteria: Common themes in bacterial glycoconjugates. Microbiol. Mol. Biol. Rev. 2014, 78, 372-417. [CrossRef] [PubMed]

2. Wiederschain, G.Y. Glycobiology: Progress, problems, and perspectives. Biochem. (Moscow) 2013, 78, 679-696. [CrossRef] [PubMed]

3. Banerjee, D.K. N-glycans in cell survival and death: Cross-talk between glycosyltransferases. Biochim. Biophys. Acta (BBA) -Gen. Subj. 2012, 1820, 1338-1346. [CrossRef] [PubMed]

4. O'Connor, S.E.; Imperiali, B. Modulation of protein structure and function by asparagine-linked glycosylation. Chem. Biol. 1996, 3, 803-812. [CrossRef]

5. Spiro, R.G. Protein glycosylation: Nature, distribution, enzymatic formation, and disease implications of glycopeptide bonds. Glycobiology 2002, 12, 43R-56R. [CrossRef] [PubMed]

6. Van Heijenoort, J. Recent advances in the formation of the bacterial peptidoglycan monomer unit (1985 to 2000). Nat. Prod. Rep. 2001, 18, 503-519. [CrossRef] [PubMed]

7. Bouhss, A.; Trunkfield, A.E.; Bugg, T.D.; Mengin-Lecreulx, D. The biosynthesis of peptidoglycan lipid-linked intermediates. FEMS Microbiol. Rev. 2008, 32, 208-233. [CrossRef]

8. Bouhss, A.; Crouvoisier, M.; Blanot, D.; Mengin-Lecreulx, D. Purification and characterization of the bacterial MraY translocase catalyzing the first membrane step of peptidoglycan biosynthesis. J. Biol. Chem. 2004, 279, 29974-29980. [CrossRef]

9. Hug, I.; Feldman, M.F. Analogies and homologies in lipopolysaccharide and glycoprotein biosynthesis in bacteria. Glycobiology 2010, 21, 138-151. [CrossRef]

10. Schäfer, A.; Thiem, J. Synthesis of novel donor mimetics of UDP-Gal, UDP-GlcNAc, and UDP-GalNAc as potential transferase inhibitors. J. Org. Chem. 2000, 65, 24-29. [CrossRef]

11. Chang, R.; Vo, T.-T.; Finney, N.S. Synthesis of the C1-phosphonate analog of UDP-GlcNAc. Carbohydr. Res. 2006, 341, 1998-2004. [CrossRef] [PubMed]

12. Hajduch, J.; Nam, G.; Kim, E.J.; Fröhlich, R.; Hanover, J.A.; Kirk, K.L. A convenient synthesis of the C-1-phosphonate analogue of UDP-GlcNAc and its evaluation as an inhibitor of $O$-linked GlcNAc transferase (OGT). Carbohydr. Res. 2008, 343, 189-195. [CrossRef]

13. Vaghefi, M.M.; Bernacki, R.J.; Hennen, W.J.; Robins, R.K. Synthesis of certain nucleoside methylenediphosphonate sugars as potential inhibitors of glycosyltransferases. J. Med. Chem. 1987, 30, 1391-1399. [CrossRef] [PubMed]

14. Stolz, F.; Blume, A.; Hinderlich, S.; Reutter, W.; Schmidt, R.R. C-Glycosidic UDP-GlcNAc analogues as inhibitors of UDP-GlcNAc 2-epimerase. Eur. J. Org. Chem. 2004, 2004, 3304-3312. [CrossRef]

15. Chen, G.-R.; Praly, J.-P. Free-radical and ionic routes towards hydrolytically stable and bioactive C-glycosyl compounds. Comptes Rendus Chim. 2008, 11, 19-28. [CrossRef]

16. Auberger, N.; Gravier-Pelletier, C.; Le Merrer, Y. Synthesis of a $\beta$-ketophosphonate bioisostere of UDP-N-acetylglucosamine. Eur. J. Org. Chem. 2009, 2009, 3323-3326. [CrossRef]

17. Grugier, J.; Xie, J.; Duarte, I.; Valéry, J.-M. Synthesis of 2-(N-Acetylamino)-2-deoxy-C-glucopyranosyl nucleosides as potential inhibitors of chitin synthases. J. Org. Chem. 2000, 65, 979-984. [CrossRef]

18. Khaled, A.; Piotrowska, O.; Dominiak, K.; Auge, C. Exploring specificity of glycosyltransferases: Synthesis of new sugar nucleotide related molecules as putative donor substrates. Carbohydr. Res. 2008, 343, 167-178. [CrossRef]

19. Tóth, M.; Somsák, L. 5'-Uridyl derivatives of N-glycosyl allophanic acid and biuret. Carbohydr. Res. 2010, 345, 163-167. [CrossRef]

20. Yeoh, K.K.; Butters, T.D.; Wilkinson, B.L.; Fairbanks, A.J. Probing replacement of pyrophosphate via click chemistry; synthesis of UDP-sugar analogues as potential glycosyl transferase inhibitors. Carbohydr. Res. 2009, 344, 586-591. [CrossRef] 
21. Babič, A.; Gobec, S.; Gravier-Pelletier, C.; Le Merrer, Y.; Pecar, S. Synthesis of 1-C-linked diphosphate analogues of UDP-N-Ac-glucosamine and UDP-N-Ac-muramic acid. Tetrahedron 2008, 64, 9093-9100. [CrossRef]

22. Kim, Y.J.; Ichikawa, M.; Ichikawa, Y. A rationally designed inhibitor of $\alpha-1,3$-galactosyltransferase. J. Am. Chem. Soc. 1999, 121, 5829-5830. [CrossRef]

23. Wang, R.; Steensma, D.H.; Takaoka, Y.; Yun, J.W.; Kajimoto, T.; Wong, C.-H. A search for pyrophosphate mimics for the development of substrates and inhibitors of glycosyltransferases. Bioorg. Med. Chem. 1997, 5, 661-672. [CrossRef]

24. Ballell, L.; Young, R.J.; Field, R.A. Synthesis and evaluation of mimetics of UDP and UDP- $\alpha$-D-galactose, dTDP and dTDP- $\alpha$-D-glucose with monosaccharides replacing the key pyrophosphate unit. Org. Biomol. Chem. 2005, 3, 1109-1115. [CrossRef]

25. Niewiadomski, S.; Beebeejaun, Z.; Denton, H.; Smith, T.K.; Morris, R.J.; Wagner, G.K. Rationally designed squaryldiamides-A novel class of sugar-nucleotide mimics? Org. Biomol. Chem. 2010, 8, 3488-3499. [CrossRef]

26. Yuan, X.; Linhardt, R.J. Recent advances in the synthesis of C-oligosaccharides. Curr. Top. Med. Chem. 2005, 5, 1393-1430. [CrossRef]

27. Compain, P.; Chagnault, V.; Martin, O.R. Tactics and strategies for the synthesis of iminosugar C-glycosides: A review. Tetrahedron: Asymmetry 2009, 20, 672-711. [CrossRef]

28. Gao, J.; Chu, X.; Qiu, Y.; Wu, L.; Qiao, Y.; Wu, J.; Li, D. Discovery of potent inhibitor for farnesyl pyrophosphate synthase in the mevalonate pathway. Chem. Commun. 2010, 46, 5340-5342. [CrossRef] [PubMed]

29. McKenna, C.E.; Rassier, M.E.; Dousa, T.P. Alpha.-Halo [(phenylphosphinyl) methyl] phosphonates as specific inhibitors of $\mathrm{Na}^{+}$-gradient-dependent $\mathrm{Na}^{+}$-phosphate cotransport across renal brush border membrane. J. Med. Chem. 1992, 35, 4885-4892. [CrossRef]

30. Gelat, F.; Lacomme, C.; Berger, O.; Montchamp, J.-L.; Gavara, L. Synthesis of (phosphonomethyl) phosphinate pyrophosphate analogues via the phospha-Claisen condensation. Org. Biomol. Chem. 2015, 13, 825-833. [CrossRef]

31. Bisseret, P.; Eustache, J. H-Phosphonylphosphonate triethylester: The first member of a novel family of stable bisphosphorylated compounds; its short synthesis and reactivity with aldehydes. Tetrahedron Lett. 2001, 42, 8451-8453. [CrossRef]

32. Fusaro, M.B.; Chagnault, V.; Josse, S.; Postel, D. Metal-free oxidative lactonization of carbohydrates using molecular iodine. Tetrahedron 2013, 69, 5880-5883. [CrossRef]

33. Xie, J.; Molina, A.; Czernecki, S. Alkylidenation of sugar lactones and further transformation to C-glycosides. J. Carbohydr. Chem. 1999, 18, 481-498. [CrossRef]

34. Kang, S.Y.; Song, K.-S.; Lee, J.; Lee, S.-H.; Lee, J. Synthesis of pyridazine and thiazole analogs as SGLT2 inhibitors. Bioorg. Med. Chem. 2010, 18, 6069-6079. [CrossRef]

35. Granier, T.; Vasella, A. Synthesis and some transformations of 2-acetamido-5-amino-3,4,6-tri-O-benzyl2,5-dideoxy-D-glucono-1,5-lactam. Helvetica Chim. Acta 1998, 81, 865-880. [CrossRef]

36. Teulade, M.-P.; Savignac, P.; Aboujaoude, E.E.; Collignon, N. Carbanions phosphonates $\alpha$-lithiés: Synthèse, basicité comparée et stabilité à l'autocondensation. J. Organomet. Chem. 1986, 312, 283-295. [CrossRef]

37. Stowell, M.H.; Witte, J.F.; Mcclard, R.W. The phosphonylphosphinyl dianion: A convenient synthon for the preparation of biologically interesting phosphonylphosphinyl (P-C-P-C) compounds. Tetrahedron Lett. 1989, 30, 411-414. [CrossRef]

38. Dondoni, A.; Marra, A.; Pasti, C. Stereoselective synthesis of C-glycosylphosphonates from their ketols. Reconsideration of an abandoned route. Tetrahedron: Asymmetry 2000, 11, 305-317. [CrossRef]

39. Yang, W.-B.; Wu, C.-Y.; Chang, C.-C.; Wang, S.-H.; Teo, C.-F.; Lin, C.-H. Facile synthesis of conjugated exo-glycals. Tetrahedron Lett. 2001, 42, 6907-6910. [CrossRef]

40. Belica, P.S.; Franck, R.W. Benzylic C-glycosides via the Ramberg-Backlund reaction. Tetrahedron Lett. 1998, 39, 8225-8228. [CrossRef]

41. Redon, S.; Wierzbicki, M.; Prunet, J. A new oxa-Michael reaction and a gold-catalysed cyclisation en route to C-glycosides. Tetrahedron Lett. 2013, 54, 2089-2092. [CrossRef]

42. Díaz, G.; Ponzinibbio, A.; Bravo, R.D. Synthesis of novel 2-deoxy- $\beta$-benzyl-C-glycosides by highly stereoand chemoselective hydrogenation of exo-glycals. Carbohydr. Res. 2014, 393, 23-25. [CrossRef] [PubMed] 
43. Kaszás, T.; Ivanov, A.; Tóth, M.; Ehlers, P.; Langer, P.; Somsák, L. Pd-catalyzed coupling reactions of anhydro-aldose tosylhydrazones with aryl bromides to produce substituted exo-glycals. Carbohydr. Res. 2018, 466, 30-38. [CrossRef]

44. Massarweh, A.; Bosco, M.; Iatmanen-Harbi, S.; Tessier, C.; Auberger, N.; Busca, P.; Chantret, I.; Gravier-Pelletier, C.; Moore, S.E.H. Demonstration of an oligosaccharide-diphosphodolichol diphosphatase activity whose subcellular localization is different than those of dolichyl-phosphate-dependent enzymes of the dolichol cycle. J. Lipid Res. 2016, 57, 1029-1042. [CrossRef] [PubMed]

45. Massarweh, A.; Bosco, M.; Chantret, I.; Léger, T.; Jamal, L.; Roper, D.I.; Dowson, C.G.; Busca, P.; Bouhss, A.; Gravier-Pelletier, C.; et al. Bacterial lipid II Analogs: Novel in vitro substrates for Mammalian Oligosaccharyl Diphosphodolichol Diphosphatase (DLODP) Activities. Molecules 2019, 24, 2135. [CrossRef]

46. Fleischer, S.; Kervina, M. Subcellular fractionation of rat liver. In Methods in Enzymology; Elsevier BV: Amsterdam, the Netherlands, 1974; Volume 31, pp. 6-41.

Sample Availability: Samples of the compound 27-29 are available from the authors via the chemical library of UMR 8601.

Publisher's Note: MDPI stays neutral with regard to jurisdictional claims in published maps and institutional affiliations.

(C) 2020 by the authors. Licensee MDPI, Basel, Switzerland. This article is an open access article distributed under the terms and conditions of the Creative Commons Attribution (CC BY) license (http://creativecommons.org/licenses/by/4.0/). 